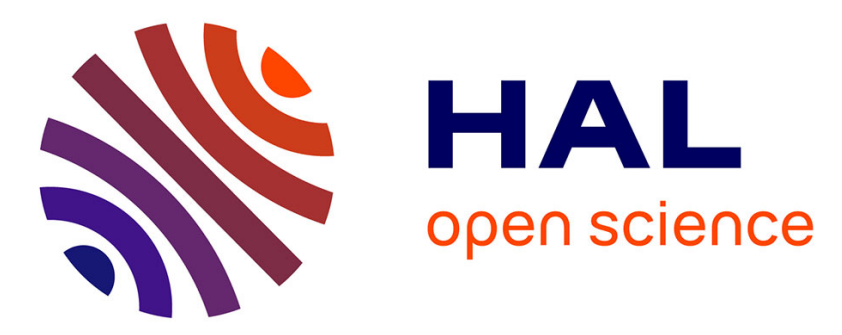

\title{
Fusion of MultiSpectral and Panchromatic Images Based on Morphological Operators
}

\author{
Rocco Restaino, Gemine Vivone, Mauro Dalla Mura, Jocelyn Chanussot
}

\section{To cite this version:}

Rocco Restaino, Gemine Vivone, Mauro Dalla Mura, Jocelyn Chanussot. Fusion of MultiSpectral and Panchromatic Images Based on Morphological Operators. IEEE Transactions on Image Processing, 2016, 25 (6), pp.2882-2895. 10.1109/TIP.2016.2556944 . hal-01303222

\section{HAL Id: hal-01303222 \\ https://hal.science/hal-01303222}

Submitted on 16 Apr 2016

HAL is a multi-disciplinary open access archive for the deposit and dissemination of scientific research documents, whether they are published or not. The documents may come from teaching and research institutions in France or abroad, or from public or private research centers.
L'archive ouverte pluridisciplinaire HAL, est destinée au dépôt et à la diffusion de documents scientifiques de niveau recherche, publiés ou non, émanant des établissements d'enseignement et de recherche français ou étrangers, des laboratoires publics ou privés. 


\title{
Fusion of MultiSpectral and Panchromatic Images Based on Morphological Operators
}

\author{
Rocco Restaino Member, IEEE, Gemine Vivone, Mauro Dalla Mura Member, IEEE, and \\ Jocelyn Chanussot Fellow, IEEE
}

\begin{abstract}
Nonlinear decomposition schemes constitute an alternative to classical approaches for facing the problem of data fusion. In this paper we discuss the application of this methodology to a popular remote sensing application called pansharpening, which consists in the fusion of a low resolution multispectral image and a high resolution panchromatic image. We design a complete pansharpening scheme based on the use of morphological half gradients operators and demonstrate the suitability of this algorithm through the comparison with state of the art approaches. Four datasets acquired by the Pleiades, Worldview-2, Ikonos and Geoeye-1 satellites are employed for the performance assessment, testifying the effectiveness of the proposed approach in producing top-class images with a setting independent of the specific sensor.
\end{abstract}

\section{INTRODUCTION}

The application of Mathematical Morphology (MM) to image processing has been experiencing a considerable success in a wide class of research fields, such as medical imaging, document processing and remote sensing [1], [2]. Classical tasks addressed with morphological approaches are texture analysis, image segmentation and classification. Several studies have also shown that MM methods are also a viable option for data fusion applications, e.g., for combining multi-focus and multi-modal images [3], [4], [5].

It is well known that by combining several images of the same scene, characterized by different conditions of acquisition, allows to access a more precise description of the imaged objects by overcoming the intrinsic limitations of each modality considered separately [6]. A key example attracting a large interest in recent years is the pansharpening, which refers to the generation of synthetic high resolution multichannel satellite images characterized by both a high spatial resolution and spectral diversity. The products of pansharpening find a widespread use in platforms such as Google Earth and Microsoft Bing, as long as base data for scientific studies [7], [8]. Two sensors acquiring a multispectral (MS) image with low spatial resolution and a PANchromatic (PAN) image with high spatial resolution are often available on board of

R. Restaino is with the Dept. of Information Eng., Electrical Eng. and Applied Math. (DIEM), University of Salerno, Via Giovanni Paolo II, 132 I-84084 Fisciano (SA), Italy. E-mail:restaino@unisa.it

G. Vivone is with the North Atlantic Treaty Organization (NATO) Science and Technology Organization (STO) Centre for Maritime Research and Experimentation, 19126 La Spezia, Italy. E-mail:vivone@cmre.nato.int

M. Dalla Mura is with GIPSA-Lab, Grenoble Institute of Technology, France. E-mail:mauro.dalla-mura@gipsa-lab.grenoble-inp.fr

J. Chanussot is with GIPSA-Lab, Grenoble Institute of Technology, France and with the Faculty of Electrical and Computer Engineering, University of Iceland. E-mail:jocelyn.chanussot@gipsa-lab.grenoble-inp.fr the same satellite platform (e.g., Quickbird, Ikonos, SPOT, Landsat) producing simultaneous acquisitions that enjoy the favorable condition of being registered. Within data fusion problems, pansharpening shows some specific characteristics, as for example, the ratios in the different spatial resolutions of the available images and the need to preserve the characteristics of the MS data. These peculiarities have caused the development of a vast dedicated scientific literature [8], composed by both classical and novel approaches. Classical approaches are in general based on relatively simple fusion schemes and are characterized by a low computational complexity [8]. Some recently proposed techniques depart from the classical architectures, such as those based on sparse representation theory [9], [10], [11], Bayesian inference [12], or variational methods [13]. However, although some of these latter approaches have shown promising results, they are still unpractical in operational scenarios due to their significant computational burden [14]. For this reason, the practical interest is still focused on classical approaches that have been well studied and assessed [8], [15].

A classical pansharpening algorithm is articulated in two successive steps: i) the extraction of the spatial details from the PAN image (that are not resolved in the MS) obtained by subtracting to the PAN a low spatial resolution version of it, and ii) the injection of the extracted details in the available MS image. Component Substitution (CS) methods generate a low resolution PAN image from a linear combination of the available MS channels. The CS name arises from the equivalence of this approach with the substitution of a component in a transformed domain, as, for example, in the space generated through the IHS (Intensity-Hue-Saturation) transformation, the Principal Component Analysis (PCA) and the Gram-Schmidt $(G S)$ orthogonalization procedure. The CS methods produce visually appealing images and are widely used for their computational efficiency and their robustness to misregistration and aliasing errors [16]. However their spectral accuracy is often low and tends to get worse with the increase of channels [8]. An alternative technique for extracting the details is provided by the MultiResolution Analysis (MRA) of the PAN image, which is generally obtained by linear decomposition methods, such as those based on wavelet [17], contourlet [18] and curvelet [19] transforms or Laplacian pyramids [20]. This approach often yields a good balance between the rendering of spatial and spectral features, especially when the design takes into account the characteristics of the MS sensor [8].

In this work we investigate non-linear MRA schemes, implemented with morphological pyramids, which are non- 
linear decomposition schemes based on morphological operators [21], [22], [23], as an alternative to the conventional linear MRA approach. Morphological pyramids have been proved their usefulness in many image processing applications, such as color image coding [24], volume rendering [25] and the fusion of medical images [4], [26], [27]. To date, only the preliminary works of Laporterie et al. [28], [29] and the contribution of Bejinariu et al. [30] exploit the morphological signal decomposition for solving the pansharpening problem. In all the aforementioned works the assessment of the algorithm has been limited to the visual analysis of the final products or to a reduced quantitative evaluation as in [28].

The choice of the morphological operator to be used in the definition of morphological pyramid is an aspect of utmost importance since it directly affects the extraction of the details from the PAN image. In this paper we study the use of a detail extraction operator based on morphological halfgradients [2], whose application to the fusion of simulated images has shown preliminary encouraging results [31]. For the sake of comparison, we extensively consider methods based on morphological decomposition schemes that were purposely proposed for data fusion, and we examine several techniques designed for the enhancement of spatial details for applications different to pansharpening. In many cases the algorithms are modified to meet the requirements of pansharpening. For example, the bias introduced by most morphological operators [32], [33], [34] represents an undesirable feature that has to be eliminated since it can cause a spectral distortion in the results. In this paper we give a physical interpretation of this approach. We show that, when using the High Pass Modulation (HPM) injection scheme [35], the proposed data fusion algorithm can be viewed as a particular instance of the contrast-preserving approaches [36], in which, according to the Weber's definition [37], the background luminance is estimated as the midrange value of neighboring pixels [38].

We fully evaluate the performance of the proposed algorithm by employing four real data sets, acquired by four different satellites, say Pleiades, Worldview-2, Ikonos and Geoeye-1. The novel approach is thus appraised in comparison to several high performance methods, belonging to the CS and the MRA categories, in a wide class of working conditions. More specifically we report here the assessment of the algorithm at the nominal resolution of the available images, confirming the results obtained in [31] with simulated data sets at reduced resolution.

The paper is organized as follows. In Sect. II we present the pansharpening problem, focusing on the solutions based on MRA schemes. In Sect. III we present a brief review of the MM concepts related to multiresolution schemes and motivate the choice of the operator used for the proposed algorithm. Sect. IV is devoted to the presentation of the results on real data: after the quantitative evaluation of the possible alternatives based on nonlinear decomposition schemes, the proposed pansharpening algorithm is compared to state of the art approaches. Concluding remarks are finally reported in Sect. V.

\section{THE PANSHARPENING PROBLEM}

The pansharpening process aims at producing a High spatial Resolution MS (HRMS) image HRMS $=\left\{\mathbf{H R M S}_{k}\right\}_{k=1, \ldots, N}$, with $N$ bands, by combining an available low spatial resolution MultiSpectral (MS) image $\mathbf{M S}=\left\{\mathbf{M S}_{k}\right\}_{k=1, \ldots, N}$ and an available high resolution PAN image $\mathbf{P}$.

A key result of the pansharpening literature [8] is that almost all classical algorithms consist in adding the detail image $\mathbf{D}=\left\{\mathbf{D}_{k}\right\}_{k=1, \ldots, N}$ extracted from the PAN image to the available MS image, upsampled to the target scale, say $\widehat{\mathbf{M S}}=\left\{\widehat{\mathbf{M S}}_{k}\right\}_{k=1, \ldots, N}$. Commonly, the PAN image $\mathbf{P}$ is firstly equalized with respect to $\mathbf{M S} \mathbf{S}_{k}$, yielding the $\mathbf{P}_{k}^{0}$ image. As a consequence, the $k$-th band of the fusion product $\widehat{\mathbf{M S}}=\left\{\widehat{\mathbf{M S}}_{k}\right\}_{k=1, \ldots, N}$ can be described by the following equation:

$$
\widehat{\mathbf{M S}}_{k}=\widetilde{\mathbf{M S}}_{k}+g_{k} \mathbf{D}_{k}=\widetilde{\mathbf{M S}}_{k}+g_{k}\left(\mathbf{P}_{k}^{0}-\mathbf{P}_{k}^{\text {low }}\right),
$$

in which $\left\{g_{k}\right\}_{k=1, \ldots, N}$ are the injection gains and $\mathbf{P}_{k}^{\text {low }}$ denotes, for each $k=1, \ldots, N$, a low resolution version of the PAN image that, in general, can be different for each band. Both $\left\{g_{k}\right\}_{k=1, \ldots, N}$ and $\left\{\mathbf{P}_{k}^{\text {low }}\right\}_{k=1, \ldots, N}$ constitute distinguishing features of the various pansharpening methods.

The most common choices for the former are given by

$$
g_{k}=1, \quad k=1, \ldots, N,
$$

leading to the additive injection scheme or High-Pass Filtering (HPF) scheme [39], [35], and

$$
g_{k}=\frac{\widetilde{\mathbf{M S}}_{k}}{\mathbf{P}_{k}^{l o w}}, \quad k=1, \ldots, N,
$$

which is called multiplicative injection scheme or High-Pass Modulation (HPM) scheme [35].

Instead, the approach used for obtaining $\mathbf{P}_{k}^{\text {low }}$ differentiates CS and MRA methods. In the former case $\mathbf{P}_{k}^{\text {low }}$ is achieved as a linear combination of the MS image channels. In MRA methods the low resolution PAN images $\mathbf{P}_{k}^{\text {low }}$ is derived from the available PAN image through a pyramidal decomposition, which consists in producing a sequence of approximations with successively reduced amount of spatial details (i.e., versions of the original image at progressively lower resolutions). For each $k=1, \ldots, N$, the procedure starts from the initial image $\mathbf{P}_{k}^{0}$ and, at each decomposition level $l$, the approximation $\mathbf{P}_{k}^{l}$ is obtained by applying the decomposition operator $T_{k}^{l}$ to the approximation at level $l-1$,

$$
\mathbf{P}_{k}^{l}=T_{k}^{l}\left[\mathbf{P}_{k}^{l-1}\right], \quad l=1,2, \ldots L .
$$

A rather general form for the decomposition operator includes the analysis operators $\psi_{k}^{l}$ and a downsampling step by a factor $R$, that we denote as $R^{\downarrow}$, namely it can be written as

$$
T_{k}^{l}=R^{\downarrow} \psi_{k}^{l}
$$

In linear pyramids the analysis operator is implemented through the convolution of the image with a low pass mask $h_{k}^{l}$, i.e., $\psi_{k}^{l}[\cdot]=h_{k}^{l} * \cdot$. Examples of analysis operators suited 
to pansharpening applications are average and Gaussian filters and wavelets [8].

Decimated or multiscale pyramids are characterized by a downsampling step with $R>1$ and thus produce approximations with smaller and smaller sizes. In that case a widely used option consists in choosing the same analysis operator at every scale, i.e., $\psi_{k}^{l}=\psi_{k}$ and in using $R=2$ (dyadic decompositions). In undecimated or single-scale pyramids no downsampling is performed (i.e., $R=1$ ) and thus the approximations have the same size at each level. Different operators are required at the various levels, in order to analyze the image with different spatial resolution.

In pansharpening algorithms described by Eq. 1 the low resolution panchromatic image $\mathbf{P}_{k}^{\text {low }}$ retains the information at the coarsest resolution and is thus obtained by upsampling (if needed) the approximation $\mathbf{P}_{k}^{L}$ to the size of the original PAN image $\mathbf{P}$. For that reason the required image is obtained according to the formula

$$
\mathbf{P}_{k}^{\text {low }}=\left(R^{\uparrow}\right)^{L} \mathbf{P}_{k}^{L},
$$

where $R^{\uparrow}$ denotes an upsampling step by a factor $R$ and $\left(R^{\uparrow}\right)^{L}$ indicates that $R^{\uparrow}$ is applied $L$ times. Note that for undecimated decomposition scheme, the upsampling step coincides with the identity operator, i.e. $R^{\uparrow}=i d$.

The objective of the pansharpening algorithms is the reconstruction of the spatial details missing in the MS image but resolved in the PAN. Therefore, the way the spatial details are extracted from the PAN image is crucial since it directly affects the rendering of the spatial information in the pansharpened image. When considering an approach based on image decomposition as the one proposed in this paper, the consistency of the spatial details extracted in the decomposition levels is a fundamental characteristic. Moreover, the preservation of the radiometric balance of the MS image across the spectral channels in the fused image is another complementary requirement. For the above-mentioned reasons, pansharpening algorithms are typically evaluated on their capability in both rendering the spatial details and avoiding spectral distortions, which are usually tested by comparison with the original PAN and MS image, respectively.

\section{NONLINEAR PYRAMIDAL SCHEMES}

Pyramidal decomposition scheme is a versatile tool for grabbing the fine spatial information contained in the panchromatic image. The focus of this work is the investigation of the alternatives offered by nonlinear operators for completing the signal decomposition, with reference to the pansharpening problem and its specific requirements.

\section{A. Basics of morphological operators}

Morphological operators act by probing a scalar image I : $E \subseteq \mathbb{Z}^{2} \rightarrow V \subseteq \mathbb{Z}$ through a set called Structuring Element (SE) $B$ [2]. The SE is defined by its spatial support $N_{B}(\mathbf{x})$ that is the neighborhood with respect to the position $\mathbf{x} \in E$ in which $B$ is centered and by its values. Flat SEs are characterized by unitary values and the only free parameters for defining $B$ are the origin and $N_{B}$.
The two basic operators are the Erosion $\varepsilon_{B}[\mathbf{I}]$ and Dilation $\delta_{B}[\mathbf{I}]$, defined, for each point $\mathbf{x} \in \mathbf{I}$, as:

$$
\varepsilon_{B}[\mathbf{I}](\mathbf{x})=\bigwedge_{\mathbf{y} \in N_{B}(\mathbf{x})} \mathbf{I}(\mathbf{y}) ; \delta_{B}[\mathbf{I}](\mathbf{x})=\bigvee_{\mathbf{y} \in N_{B}(\mathbf{x})} \mathbf{I}(\mathbf{y}),
$$

in which $\bigwedge_{S}$ and $\bigvee_{S}$ denote the infimum and supremum value within the set $S$, respectively. The application of an erosion (resp. dilation) has as filtering effect the suppression of bright (resp. dark) regions smaller than $B$ and the enlargement of dark (resp. bright) ones. The concepts of bright and dark regions refer to the local contrast in the sense that a region has intensity values greater or lower with respect to the surrounding ones, respectively. For convenience, we introduce also Opening and Closing that correspond to the two possible sequential compositions of erosion and dilation:

$$
\gamma_{B}[\mathbf{I}]=\delta_{\breve{B}}\left[\varepsilon_{B}[\mathbf{I}]\right], \phi_{B}[\mathbf{I}]=\varepsilon_{\breve{B}}\left[\delta_{B}[\mathbf{I}]\right],
$$

with $\breve{B}$ denoting the SE obtained by reflecting $B$ with respect to its origin. An opening removes bright regions smaller than $B$ whereas a closing suppresses dark ones.

A number of morphological operators can be obtained by combining these four elementary bricks (see for example [1], [2]). In the following we focus on the operators aimed to extract the image details, or equivalently, on their complementary operators that reduce the resolution, thus representing possible candidates for the MRA analysis steps.

The internal gradient $\rho_{B}^{-}[\mathbf{I}]=\mathbf{I}-\varepsilon_{B}[\mathbf{I}]$ and the external gradient $\rho_{B}^{+}[\mathbf{I}]=\delta_{B}[\mathbf{I}]-\mathbf{I}$ are the residuals of the application of erosion and dilation.

Analogously, the top-hat transforms are the complementary operators of opening and closing, hence showing the residuals of the filtering:

$$
\begin{aligned}
\mathrm{WTH}_{B}[\mathbf{I}] & =\mathbf{I}-\gamma_{B}[\mathbf{I}], \\
\mathrm{BTH}_{B}[\mathbf{I}] & =\phi_{B}[\mathbf{I}]-[\mathbf{I}],
\end{aligned}
$$

in which WTH and BTH are the acronym of White Top Hat and Black Top Hat, respectively.

Finally the alternate use of erosion and dilation switched by a decision function is called Toggle Contrast (TC) mapping [2]:

$$
\mathrm{TC}_{B}[\mathbf{I}]=\left\{\begin{array}{lc}
\delta_{B}[\mathbf{I}], & \text { if } \rho_{B}^{+}[\mathbf{I}]<\rho_{B}^{-}(\mathbf{I}) \\
\varepsilon_{B}[\mathbf{I}], & \text { otherwise. }
\end{array}\right.
$$

The result of a TC mapping is obtained by selectively toggling between the result of a dilation or erosion according to the result which is closest to the original image. TC mapping has been useful for image sharpening [2].

\section{B. Some existing nonlinear analysis operators}

Nonlinear multiresolution schemes constitute a wide class of signal decompositions [21], [22], [23] that allow for an accurate extraction of the details from an image, which is a fundamental aspect of pansharpening. The crucial choice for their implementation is the definition of the analysis operator, which determines how the reduction of the resolution is obtained among levels. Several choices are available in the data fusion literature and others can be easily derived from other 
similar applications. In the following we present the analysis operators $\psi$ that have been object of investigation during the current study and indicate by $\bar{\psi}=i d-\psi$ the complementary operator that coincides with the detail extraction operator (except for the upsampler present in decimated schemes).

Morphological decomposition schemes were firstly proposed in [40], exploiting the use of a morphological filter, i.e., an idempotent and increasing operator [1]. In particular, the analysis operator was constituted by the sequence of an opening and closing ( $\mathrm{CO})$, namely it was defined as:

$$
\psi_{\mathrm{CO}, B}=\phi_{B} \gamma_{B}
$$

Similarly to many other elementary morphological operators, $\psi_{\mathrm{CO}, B}$ introduces a bias on the image values [32], [33], [34], i.e., the mean value of the filtered image is not preserved with respect to the original image. This constitutes a critical aspect for pansharpening application, since it can prejudice the quality of the final product. A solution to this problem consists in combining multiple operators in order to reduce the shift effect [41]. Taking advantage of the opposite bias entailed by the two morphological filters $\phi_{B} \gamma_{B}$ and $\gamma_{B} \phi_{B}$ [34], the Linear Combination of $O C$ and $C O$ (LOCO) operator defined as

$$
\psi_{\mathrm{LC}, B}=0.5\left(\phi_{B} \gamma_{B}+\gamma_{B} \phi_{B}\right)
$$

was proposed in [41] and there used for denoising applications.

The same rationale motivated the choice of Laporterie et al. [42], [43], which employed the semi-sum of opening and closing

$$
\psi_{\mathrm{TH}, B}=0.5\left(\phi_{B}+\gamma_{B}\right) .
$$

This operator is also known as pseudomedian filter [41] and is closely related to top-hats. Indeed, its complementary operator $\bar{\psi}_{\mathrm{TH}, B}$ can be easily rewritten as $\bar{\psi}_{\mathrm{TH}}=0.5\left(\mathrm{WTH}_{B}-\mathrm{BTH}_{B}\right)$ and has been widely used for enhancing the contrast of the images [2].

Analogously to top-hat transforms, the toggle contrast mapping defined in Sect. III-A can be used to implement a pyramidal decomposition scheme. In particular Bai et al. [44] constructed a detail extraction operator as the difference of the Dilation Toggle Contrast operators DTC $B_{B}=\max \left(\mathrm{TC}_{B}-\right.$ $i d, 0)$ and the Erosion Toggle Contrast operators ETC $_{B}=$ $\max \left(i d-\mathrm{TC}_{B}, 0\right)$, namely the analysis operator was defined according to the formula:

$$
\begin{aligned}
\psi_{\mathrm{TC}, B} & =i d-\bar{\psi}_{\mathrm{TC}}=i d-0.5\left[\mathrm{DTC}_{B}-\mathrm{ETC}_{B}\right] \\
& =i d-0.5\left[\max \left(\mathrm{TC}_{B}-i d, 0\right)-\max \left(i d-\mathrm{TC}_{B}, 0\right)\right] .
\end{aligned}
$$

Note explicitly that the factor 0.5 was not present in the original paper [44], but is required by the specific pansharpening application in order to preserve the dynamical range of the details.

In a successive paper Bai et al. proposed to enforce the detail extraction capability by joining the effects of top-hats and toggle contrast operators [5]. Thus, the analysis operator, that we indicate with the subscript $T T$, assumed the expression

$$
\psi_{\mathrm{TT}, B}=i d-0.5\left[\left(\mathrm{WTH}_{B}-\mathrm{BTH}_{B}\right)-\left(\mathrm{DTC}_{B}-\mathrm{ETC}_{B}\right)\right],
$$

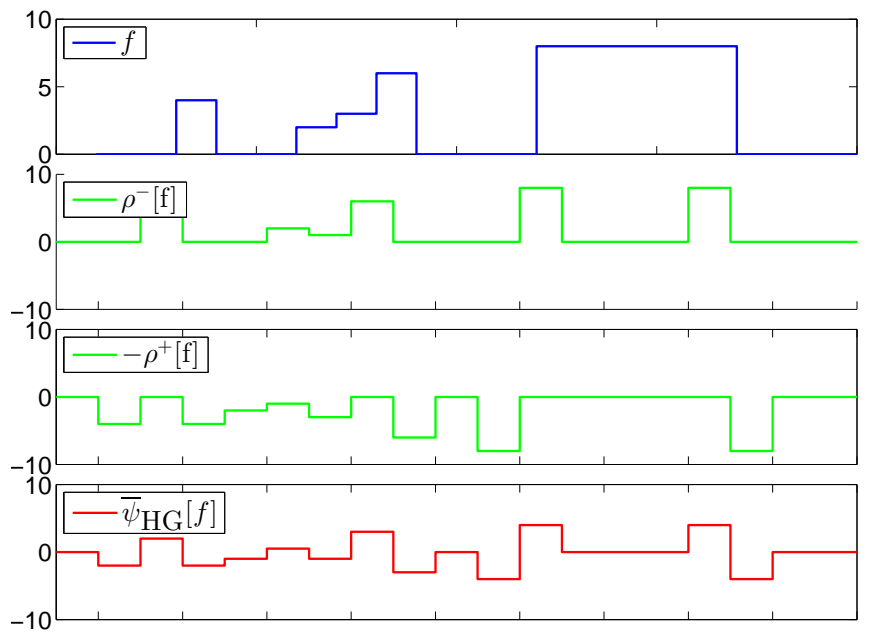

Fig. 1: Examples of application of morphological gradients obtained through a flat SE with $N_{B}=\{-1,0,1\}$, to a onedimensional function $f$ : (a) function $f$, (b) internal gradient $\rho^{-}=f-\epsilon_{B}[f]$; (c) external gradient $\rho^{+}=\delta_{B}[f]-f$; (d) proposed analysis operator $\psi_{\mathrm{HG}}=0.5\left(\rho^{-}-\rho^{+}\right)=0.5(f-$ $\left.\epsilon_{B}[f]\right)+0.5\left(f-\delta_{B}[f]\right)$.

in which, for the same reason, we added again the factor 0.5 with respect to the original definition.

Finally we report a different example of nonlinear decomposition scheme. The Multiscale Median Transform (MMT) is a very simple instance proposed in [45] for astronomical applications. The analysis operator is defined as

$$
\psi_{\mathrm{ME}, B}=\operatorname{Med}_{B}
$$

with $\mathrm{Med}_{B}$ denoting a median filter with support $N_{B}$. We use the subscript $B$ for indicating that the median value is calculated on the pixel neighborhood $N_{B}$, for uniformity of notation.

\section{Analysis operator based on half-gradients}

In this work we propose to use the half gradients for detail extraction. Fig. 2(b) and Fig. 2(c) illustrate the effect of the internal and external gradients with a flat SE $B$ with neighborhood $N_{B}=\{-1,0,1\}$ on the piecewise-constant monodimensional signal $f$ depicted in Fig. 2(a). In the presence of discontinuities both half-gradients assume positive values that constitutes an approximation of the norm of the signal gradient [2]. The positive values of the internal gradient $\rho^{-}[f]$ follow the positive discontinuities and precede the negative ones, while those of $\rho^{+}[f]$ experience the opposite behavior. As a result, the difference of the two gradients represents a suitable detail extraction operator $\bar{\psi}_{\mathrm{HG}, B}$, since it reproduces the variations of the function with respect to the local mean. In particular we use the following definition

$$
\bar{\psi}_{\mathrm{HG}, B}=0.5\left(\rho^{-}-\rho^{+}\right)=0.5\left(i d-\epsilon_{B}\right)-0.5\left(\delta_{B}-i d\right),
$$

in which the factor 0.5 is applied to preserve the property of approximating the image gradient norm. 
The corresponding analysis filter is given by

$$
\begin{aligned}
\psi_{\mathrm{HG}, B} & =i d-\bar{\psi}_{\mathrm{HG}, B} \\
& =i d-\left[0.5\left(i d-\epsilon_{B}\right)-0.5\left(\delta_{B}-i d\right)\right] \\
& =0.5\left(\epsilon_{B}+\delta_{B}\right),
\end{aligned}
$$

namely, it corresponds to the semi-sum of dilation and erosion. According to the definitions reported in Eqs. (7), $\psi_{H G}$ calculates the semi-sum of the minimum and the maximum value (or the midrange [38]) of the function within the neighborhood $N_{B}$.

This remark is particularly significant for pansharpening methods based on the HPM injection method. In this case the fusion equation (1) specifies as

$$
\widehat{\mathbf{M S}}_{k}=\widetilde{\mathbf{M S}}_{k}+\widetilde{\mathbf{M S}}_{k} \frac{\mathbf{P}_{k}^{0}-\mathbf{P}_{k}^{\text {low }}}{\mathbf{P}_{k}^{\text {low }}}
$$

and can be put in strong relation with the local contrast of the PAN image [36]. Indeed, using the Weber's definition of contrast [37]

$$
C_{W}=\frac{L-L_{b}}{L_{b}}
$$

in which $L$ is the pixel luminance and $L_{b}$ the background luminance, Eq. (22) can be rewritten as

$$
\widehat{\mathbf{M S}}_{k}=\widetilde{\mathbf{M S}}_{k}\left(1+C_{W}\right),
$$

where the low pass version of the PAN image acts as the background luminance. Therefore the application of $\psi_{\mathrm{HG}, B}$ corresponds to estimate the background luminance as the midrange values within a neighborhood set by the SE $B$. This approach has been already employed in [46] and is particularly advisable when small neighborhoods are considered. Indeed the midrange is a very efficient and robust estimator of the location parameter of platykurtic (with broad curvature) distributions [47], [48]. The latter are characterized by a small value $(\leq 2.2)$ of the normalized fourth central moment of the distribution, or kurtosis $K$. For instance, the midrange is the optimal estimator in the case of a uniform distribution ( $K=1.8$ ); in fact its variance decays as $1 / N^{2}$, while that of the sample mean as $1 / N$ [49]. We tested the adequacy of the platykurtic assumption by examining the distribution of the PAN values standardized with respect to the mean and the standard deviation calculated in given neighborhoods $N_{B}$. For all the available images the condition $K \leq 2.2$ was largely verified for neighborhoods containing up to 6 pixels.

\section{Effect of the detail extraction operators: A toy example}

With the aim of illustrating the main features of the different definitions, we report in Fig. 2 the results of applying the operators described in Sect. III-B and the proposed method presented in Sect. III-C to the one dimensional signal depicted in Fig. 2(a). On the left column plots the effect of the analysis operators $\psi$ is drawn with red dash lines together with the original signal $f$ (in solid blue lines); on the right column the plots report the output of the details extraction operators $\bar{\psi}=$ $i d-\psi$ (solid red lines). We implement all the morphological operators by employing a flat SE with neighborhood $N_{B}=$
$\{-1,0,1\}$ that is also used for computing the output of the median filter.

The test signal has been designed to present both a fast dynamic (first half of its domain) and slower variations (second half). In the second part the function discontinuities are spaced by intervals of constant values with length greater than the support of the SE $B$. The comparison of the operators' behavior over the whole domain evidences that in general $\bar{\psi}_{\mathrm{HG}}$ is able to extract the most significant amount of details. We underline that this is a very important feature for image fusion since smaller details in the PAN might appear as intensity variations with a spatial support of few pixels. Remarkably, all the discontinuities of the test signal are detected by the proposed operator. Moreover, the amplitude of signal variations is correctly extracted by the operator allowing the preservation of the dynamic of the signal. We will see in the following that in pansharpening this is a necessary feature to limit spectral distortions in the results. Among the other operators, the TC defined by Eq. (15) is less sensitive to the local signal variations, being zero almost everywhere. On the contrary, $\bar{\psi}_{\mathrm{LC}}$ and $\bar{\psi}_{\mathrm{TH}}$ (and the very similar $\bar{\psi}_{\mathrm{TT}}$ ) are able to reproduce the details with sufficient accuracy, but only in the first half of the observation interval, where the signal varies over intervals smaller than the SE length. In fact, in the second half of the signal support only $\bar{\psi}_{\mathrm{HG}}$ is able to detect the signal variation, while the output of the others is identically null.

\section{EXPERIMENTAL RESULTS}

In this section we compare the behavior of the different analyzed algorithms on real images. Due to the unavailability of the target HRMS image, the assessment of pansharpening algorithms is typically performed with two companion protocols [8]. The reduced resolution assessment involves the degradation of both the original MS and PAN images. This procedure has to be completed according to the Wald protocol [50], namely the MS image has to be degraded by filtering through a system mimicking the response of the sensors and by decimating by a factor equal to the resolution ratio $r$. We employed a Gaussian filter with the same gain at the Nyquist frequency of the specific sensor for the MS images and an almost ideal filter for the PAN image [51]. This methodology allows the exploitation of a wide range of quality indexes, since the original MS image acts as the target image for the fusion process. We selected the Spectral Angle Mapper (SAM) [52] as a measure of the spectral quality and two comprehensive indexes, the $\mathrm{Q} 2^{n}$-index [53], [54], and the Erreur Relative Globale Adimensionnelle de Synthèse $(E R G A S)$ [55] for evaluating both spatial and spectral quality. Optimal values are 0 for the SAM and the ERGAS and 1 for the $\mathrm{Q} 2^{n}$. On the other hand, the reduced resolution assessment is based on the scale invariance assumption that cannot be undoubtedly assumed in the real practice. For that reason it has to be supplemented by the full resolution assessment that employs the available images at the original resolution. Since the target HRMS image does not exists at that level, indexes with no reference have to be employed. In this work we separately assess the spectral and spatial quality of the 

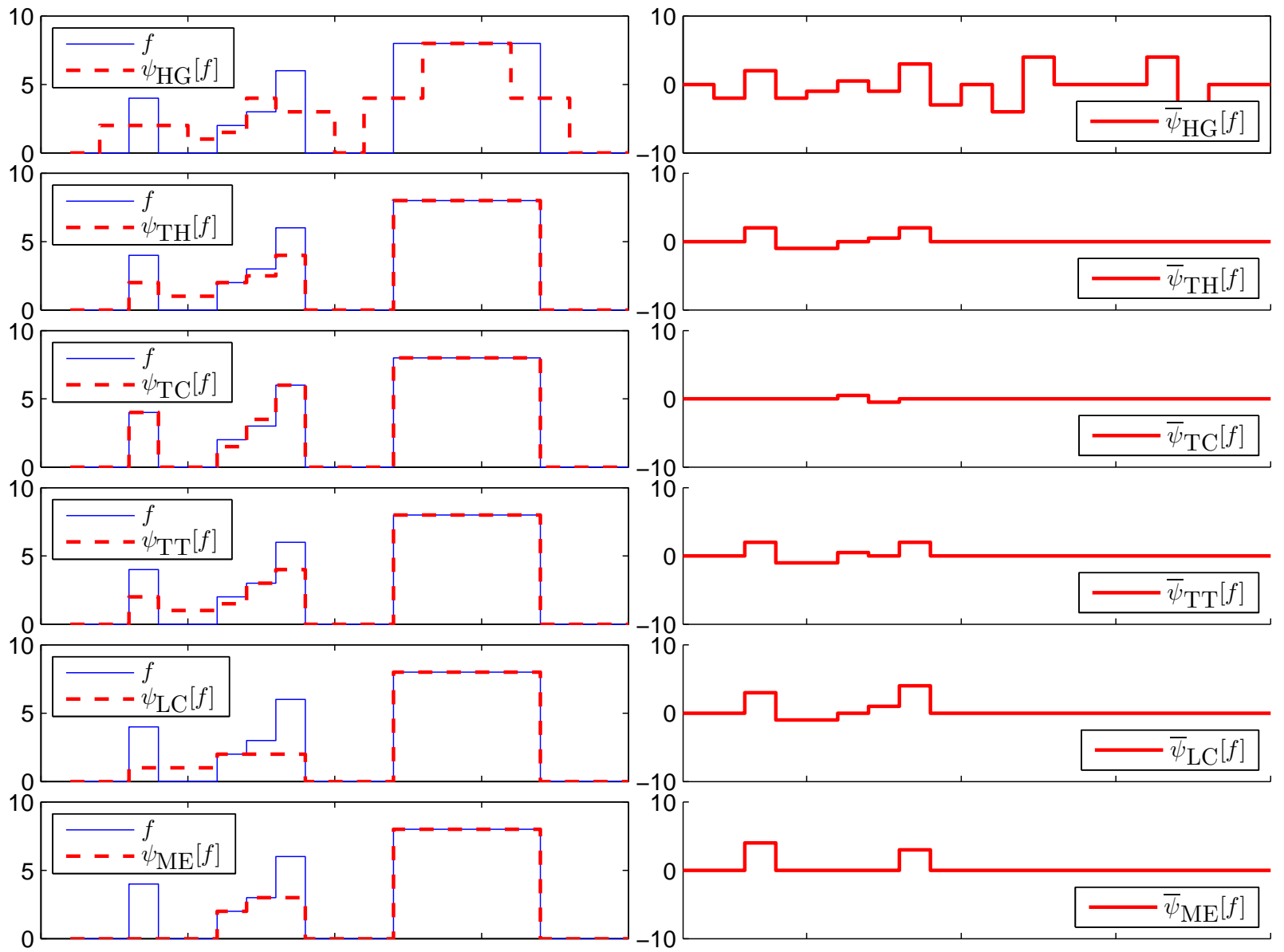

Fig. 2: Examples of morphological analysis operators (left column) and corresponding detail extraction operators (right column), based on a flat SE with $N_{B}=\{-1,0,1\}$ : from top to bottom: Half Gradients (HG), Top-Hat (TH), Toggle Contrast (TC), Top-Hat + Toggle (TT) [5], LOCO (LC) and Median (ME).

pansharpened image, by employing the $S A M$ index for the former and the Spatial Correlation Coefficient (SCC) [56] for the latter. The optimal value of the SCC index is 1 .

The employed data sets are described in the first subsection, while the performance assessment of the algorithms are reported in the following subsections. We firstly compare the different analysis operator that are eligible for implementing the morphological pyramid decomposition and then evaluate the chosen scheme together with some of the most credited algorithms present in the literature.

\section{A. Datasets}

We employ the four data sets illustrated in Fig. 3 for assessing the performance of the proposed algorithm. They are characterized by the same radiometric resolution of 11-bits and resolution ratio $r=4$, but were acquired by four different platforms, namely Pléiades, WorldView-2 (WV-2), Ikonos and Geoeye-1, whose name is used for denote the different data sets. We use the Pléiades and WorldView-2 (WV-2) data sets for the evaluation of the algorithms at reduced resolution and the Ikonos and Geoeye-1 data sets for the comparison at full resolution.
The Pléiades data set (Figs. 3(a) and 3(e)) is well-known since it was used for the 2006 contest [15] and for the reference pansharpening review [8]. It consists of four MS bands with size of $1024 \times 1024$ and resolution of $60 \mathrm{~cm}$, acquired on an urban area of Toulouse (France) by an aerial CNES platform. Due to the unavailability of the panchromatic sensor the PAN image was synthetically obtained from the MS images [15].

The WV2 data set (Figs. 3(b) and 3(f)) is composed by a MS image with eight channels (red, green, blue, and nearinfrared 1, coastal, yellow, red edge, and near-infrared 2) and a PAN image collected on an urban area of Rome (Italy). The PAN image size is $300 \times 300$ pixels and the spatial resolution of the PAN and MS images is $0.5 \mathrm{~m}$ and $2 \mathrm{~m}$, respectively.

The images of the Ikonos data set (Figs. 3(c) and 3(g)) are related to another urban area of the city of Toulouse (France). The IKONOS sensor acquires four bands (blue, green, red and near infrared) in the visible and near infrared range, together with a panchromatic channel. The spatial resolution is $4 \mathrm{~m} \times 4 \mathrm{~m}$ for the multispectral bands and $1 \mathrm{~m} \times 1 \mathrm{~m}$ for the panchromatic channel and the employed PAN image is composed by $512 \times 512$ pixels.

The Geoeye data set (Figs. 3(d) and 3(h)) was acquired over Hobart, Australia, and consists of a high resolution PAN 


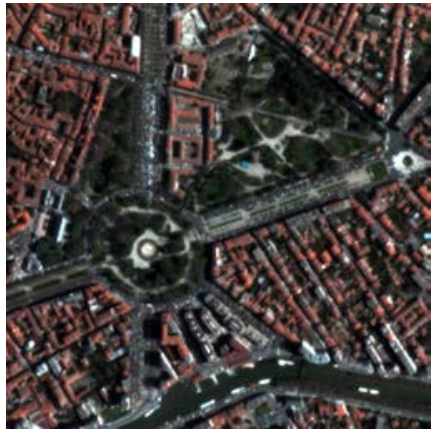

(a) Pleiades MS

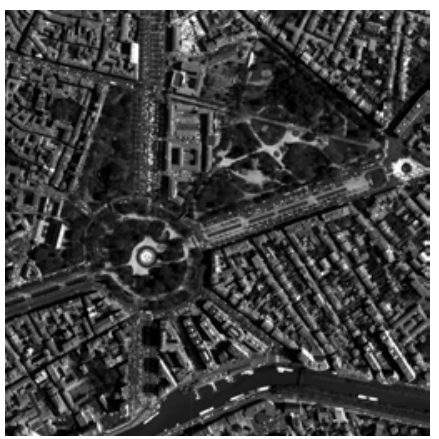

(e) Pleiades PAN

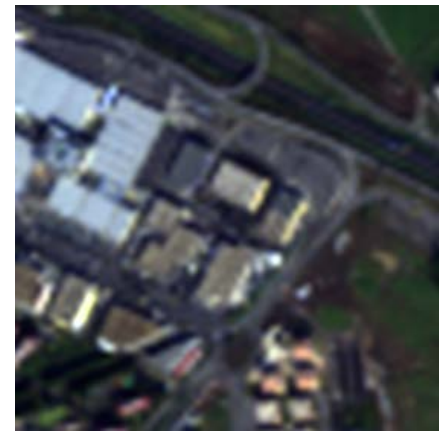

(b) WV2 MS

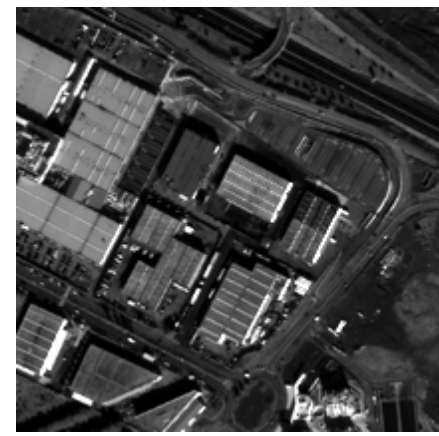

(f) WV2 PAN

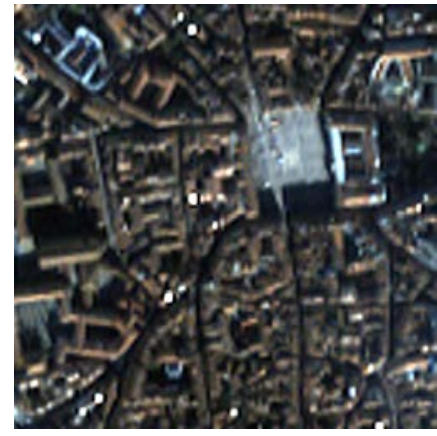

(c) Geoeye MS

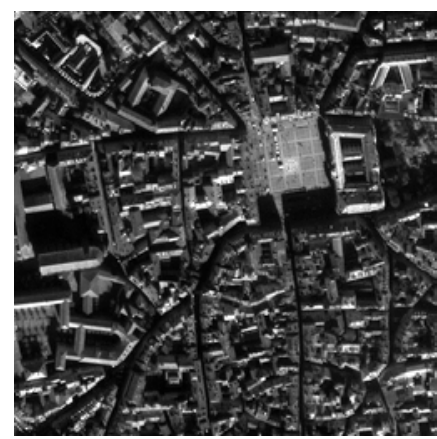

(g) Geoeye PAN

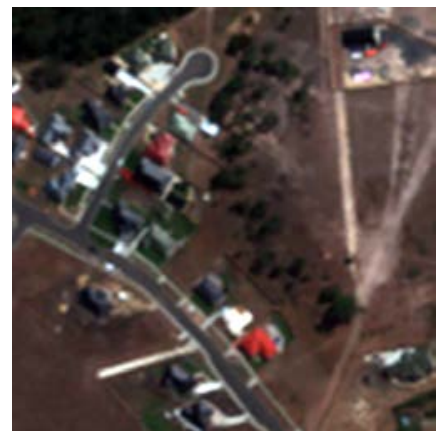

(d) Ikonos MS

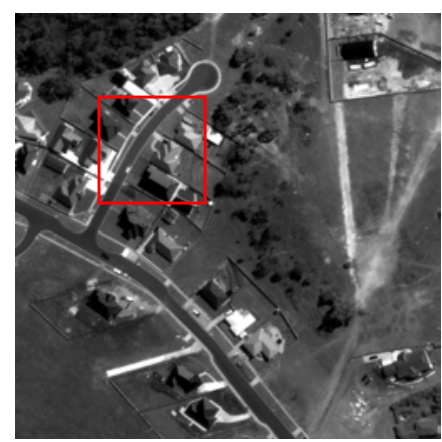

(h) Ikonos MS

Fig. 3: Data sets from left to right: (a,e) Pleiades; (b,f) WV2; (c,g) Ikonos; (d,h) Geoeye. The PAN sizes are $1024 \times 1024$, $300 \times 300,512 \times 512,512 \times 512$, respectively.

image and a four-channel MS bands (blue, green, red and near infrared channels). The data set was provided by Geoeye ${ }^{1}$ and is characterized by a spatial resolution of $0.5 \mathrm{~m}$ and $2 \mathrm{~m}$ pixels for the PAN and MS image, respectively. The PAN size is $512 \times 512$.

\section{B. Comparison of the morphological operators}

The first outcome of the investigation of real data is a visual comparison of the details extracted by the nonlinear operators described in Sects. III-B and III-C. We employed the PAN image belonging to the Geoeye data set and in particular we focus on the area delimited by the red rectangle in Fig. 3(h). The operators exploited the same SE $B$ (whose neighborhood $N_{B}$ was also used for the MMT). In particular we selected a $3 \times 3$ disk-shaped SE that was shown to achieve the best results in a preliminary study [31]. Its use has been also suggested in [24] since its cross-type structure prevents to privilege a particular direction, avoiding, at the same time, block-type effects typical of square SEs.

Even though the test does not constitute a quantitative analysis, Figs. 4(a-f) allow to confirm that $\bar{\psi}_{H G}$ is able to extract more information, yielding a defined detail image, as it was argued in the analysis of one dimensional signals described in Sect. III-D. We further apply an edge extraction operator $E$ based on the Canny method [57] to highlight the differences among the methods. Figs. 4(g-l) demonstrate that the proposed operator is able to detect a greater number of

${ }^{1}$ Geoeye: Geoeye-1 Geo ${ }^{\mathrm{TM}} 11$ bit $0.5 \mathrm{~mm}+2.0$ Bundle - Hobart Aust 1 , 02/05/2009(2009). object contours, which are also reproduced with an appreciable continuity.

The capability of extracting the shape of the objects with a remarkable accuracy is a major feature of the methods based on MM that has fostered their success in many applications, as for example noise filtering and segmentation [2]. For that reason we extend this test also to some linear filters that are widely used in pansharpening [51]. We compare the details and the edges extracted by the proposed operator with the complementary operator $\bar{\psi}_{G F}[f]=i d-\psi_{G F}[f]$ of a Gaussian low pass Filter $\psi_{G F}$ matched to the MTF of the Geoeye-1 PAN sensor (namely with gain at the Nyquist frequency $\left.G_{N y q}=0.16\right)$ and with the complementary operator $\bar{\psi}_{A T}[f]=i d-\psi_{A T}[f]$ of the Starck and Murtagh (S\&M) filter $\left.\psi_{A T}\right)$ [58] that is used in the 1-D "à trous" implementation of the wavelet decomposition [59]. Although the linear methods (and in particular the one based on the GF) reproduces a more intelligible detail image (see Fig. 4(g-h)), the application of the Canny operator shows that the morphological filter permits a more accurate detection of the contours.

Actually, the choice of the most suitable morphological operator has to be supported by a quantitative analysis on the field. For this reason the second experiment is dedicated to the assessment of a MRA-based pansharpening scheme, based on the cited nonlinear operators. We fixed the algorithm architecture by employing a decimated/interpolated decomposition exploiting dyadic subsampling and bilinear interpolation in the analysis and synthesis phase, respectively. The number $L$ of decomposition levels is set to $L=\log _{2}(r)$, where $r$ is the resolution ratio between the MS and the PAN image (in our 


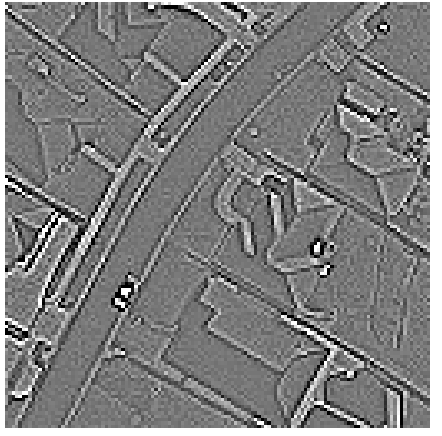

(a) $\bar{\psi}_{\mathrm{HG}}[\mathbf{P}]$

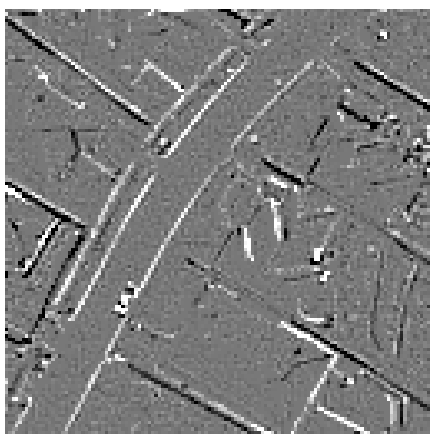

(e) $\bar{\psi}_{\mathrm{LC}}[\mathbf{P}]$

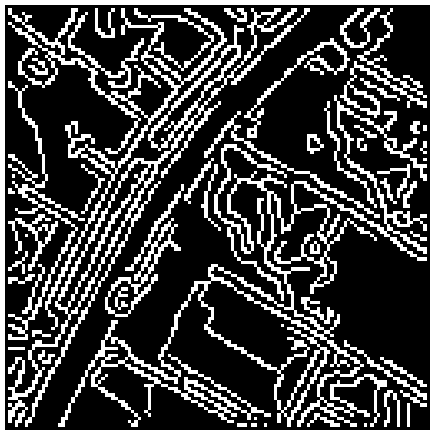

(i) $E \bar{\psi}_{\mathrm{HG}}[\mathbf{P}]$

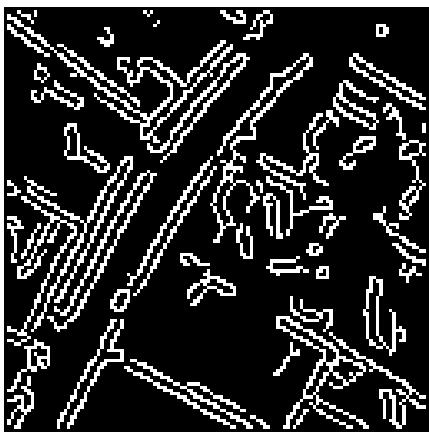

(m) $E \bar{\psi}_{\mathrm{LC}}[\mathbf{P}]$

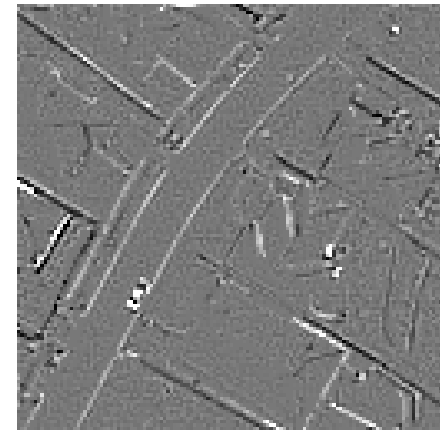

(b) $\bar{\psi}_{\mathrm{TH}}[\mathbf{P}]$

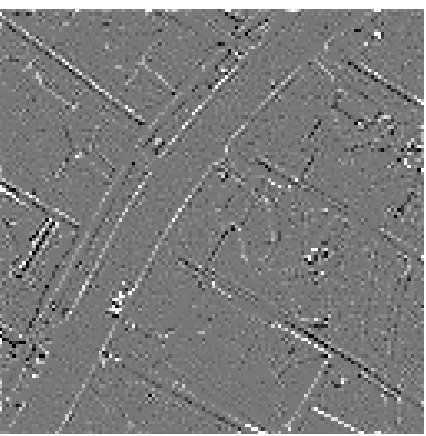

(f) $\bar{\psi}_{\mathrm{ME}}[\mathbf{P}]$

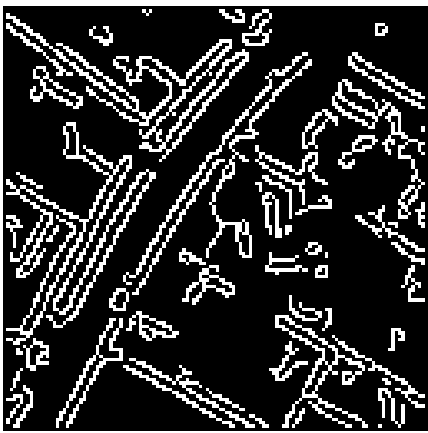

(j) $E \bar{\psi}_{\mathrm{TH}}[\mathbf{P}$

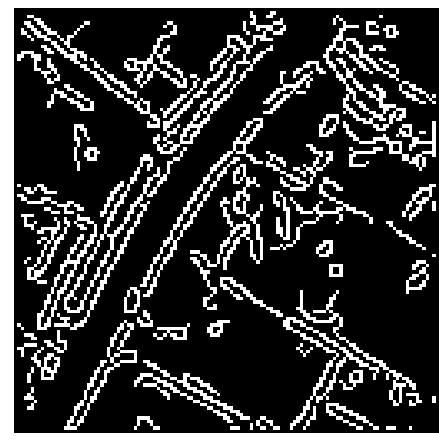

(n) $E \bar{\psi}_{\mathrm{ME}}[\mathbf{P}]$

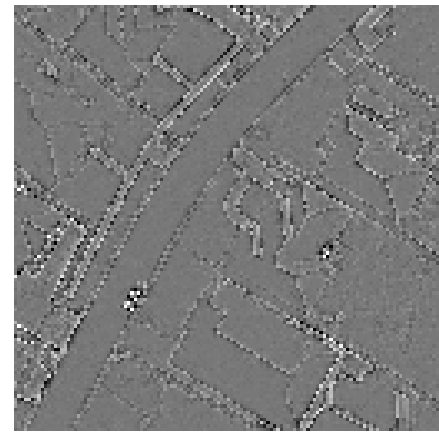

(c) $\bar{\psi}_{\mathrm{TC}}[\mathbf{P}]$

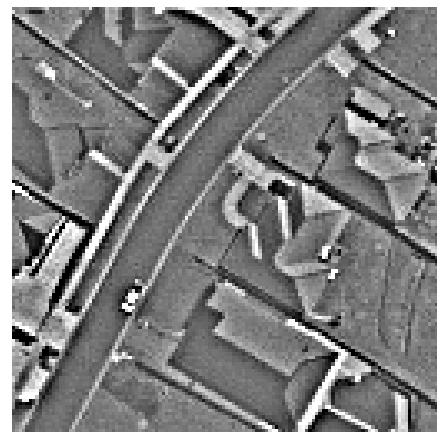

(g) $\bar{\psi}_{\mathrm{GF}}[\mathbf{P}]$

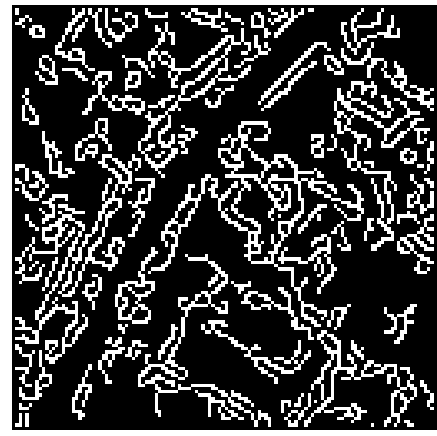

(k) $E \bar{\psi}_{\mathrm{TC}}[\mathbf{P}]$

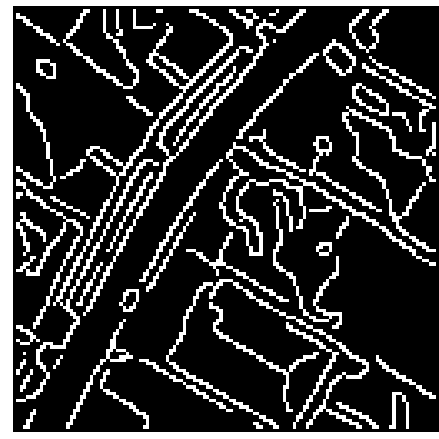

(o) $E \bar{\psi}_{\mathrm{GF}}[\mathbf{P}]$

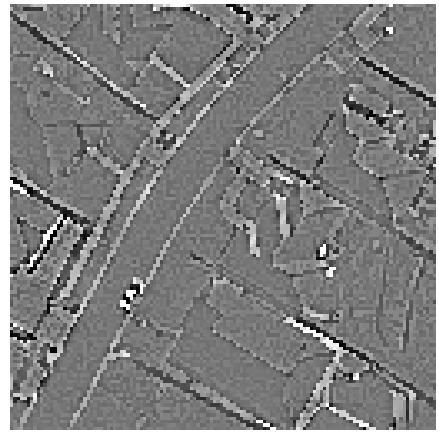

(d) $\bar{\psi}_{\mathrm{TT}}[\mathbf{P}]$

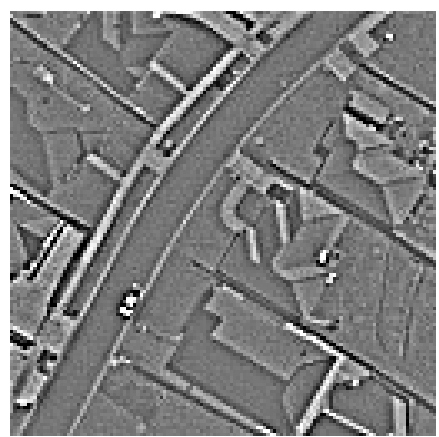

(h) $\bar{\psi}_{\mathrm{AT}}[\mathbf{P}]$

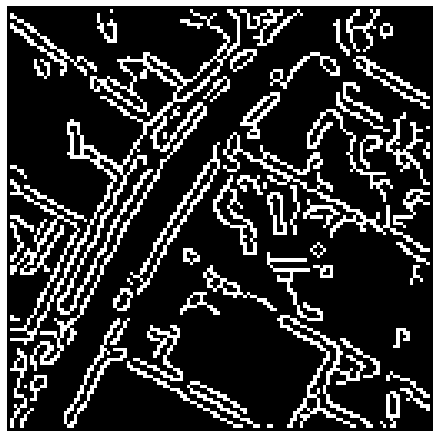

(1) $E \bar{\psi}_{\mathrm{TT}}[\mathbf{P}]$

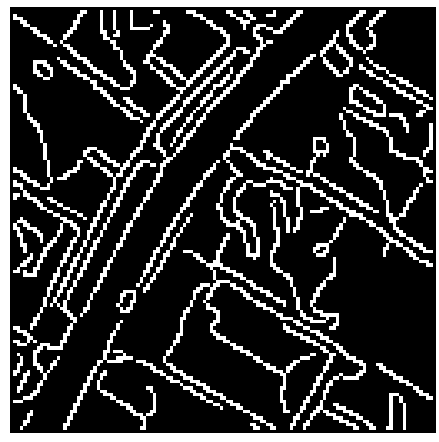

(p) $E \bar{\psi}_{\mathrm{AT}}[\mathbf{P}]$

Fig. 4: Application of the detail extraction operators and of the Canny edge detector to the area highlighted by the red rectangle in the Geoeye data set (Fig. 3(h)): (a-h) details extracted by the different morphological operators, by the Gaussian Filter (GF) and by the "à trous" algorithm (AT); (g-l) corresponding detected edges.

tests for all data sets $r=4$ and thus $L=2$ ). The details were injected according to the HPM method expressed by Eq. 3. The tested algorithms thus differ only for the MRA decomposition operator

$$
T_{\mathrm{XX}}=2^{\downarrow} \psi_{\mathrm{XX}}
$$

where $\mathrm{XX}$ indexes the adopted analysis operator by ranging 
TABLE I: QUANTITATIVE RESULTS OBTAINED BY USING THE DIFFERENT DECOMPOSITION OPERATORS TXX WITH $3 \times 3$ DIAMOND-SHAPED SE, DYADIC DECOMPOSITION AND BILINEAR INTERPOLATION FOR THE EXPANSION. FOR EACH DATASET, THE BEST RESULT AMONG OPERATORS IS MARKED IN BOLD, THE SECOND ONE IS UNDERLINED AND THE THIRD IS WRITTEN IN ITALIC CHARACTERS.

\begin{tabular}{|c|c|c|c|c|c|c|c|c|c|c|}
\hline & \multicolumn{6}{|c|}{ Reduced resolution } & \multicolumn{4}{|c|}{ Full resolution } \\
\hline & \multicolumn{3}{|c|}{ Pleiades dataset } & \multicolumn{3}{|c|}{ WV2 dataset } & \multicolumn{2}{|c|}{ Ikonos dataset } & \multicolumn{2}{|c|}{ Geoeye dataset } \\
\hline & Q4 & SAM & ERGAS & Q8 & SAM & ERGAS & SAM & SCC & SAM & SCC \\
\hline$T_{T H}$ & 0.9475 & 4.1691 & 3.5730 & 0.8935 & 3.9691 & 3.7878 & 1.6438 & 0.8316 & 1.2515 & 0.9467 \\
\hline$T_{T C}$ & 0.9305 & 4.1639 & 3.8519 & 0.8723 & 4.0576 & 3.9235 & 1.7059 & 0.8262 & 1.2724 & 0.9342 \\
\hline$T_{T T}$ & $\underline{0.9491}$ & $\underline{4.1284}$ & 3.4937 & $\underline{0.8951}$ & $\underline{3.9447}$ & 3.6673 & $\underline{1.5710}$ & $\underline{0.8696}$ & $\underline{1.2504}$ & $\underline{0.9469}$ \\
\hline$T_{L C}$ & 0.9437 & 4.1706 & 4.1003 & 0.8806 & 4.0066 & 4.4131 & 1.8183 & 0.8012 & 1.2659 & 0.9425 \\
\hline$T_{M E}$ & 0.9446 & 4.1908 & 3.6040 & 0.8899 & 4.0188 & 3.8625 & 1.6614 & 0.8152 & 1.2558 & 0.9444 \\
\hline$T_{H G}$ & 0.9503 & 4.0258 & 3.2933 & 0.9014 & 3.8773 & 3.2884 & 1.3970 & 0.9108 & 1.2408 & 0.9499 \\
\hline
\end{tabular}

in the set $\{\mathrm{TH}, \mathrm{TC}, \mathrm{TT}, \mathrm{LC}, \mathrm{ME}, \mathrm{HG}\}$.

Table I reports the values of the adopted quality indexes for the reduced and full resolution assessment protocols. The superiority of the proposed operator based on half gradients is evident in all data set and for all the indexes. The algorithm is thus able to produce images with both better spectral accuracy and huger details' content, as it is particularly evident by the values of the full resolution indexes, which separately assess the two features. Also the algorithms employing the top-hat transform, namely $T_{\mathrm{TH}}$ and $T_{\mathrm{TT}}$, allows to obtain images with appreciable quality.

\section{Comparison to pansharpening literature}

After the results obtained through the analysis reported in Sect. IV-B, a dyadic MRA pansharpening scheme using the HPM injection method and the decomposition operator

$$
T_{\mathrm{HG}}=2^{\downarrow} \psi_{\mathrm{HG}}
$$

constitutes the proposed algorithm and will be henceforth denoted as MF-HG. In this section we compare its performance to those achievable to the (few) existing pansharpening approaches based on morphological operators and to the principal classical approaches belonging to both the CS and MRA classes. As a reference, we further reports the results obtained by using the same algorithm without the morphological analysis filter. In other words the approach employs the decomposition operator

$$
T_{1}=2^{\downarrow} i d
$$

and will denoted as MF-1, since it also corresponds to the use of a morphological operator with SE of size 1.

The pansharpening literature has not been populated by many methods employing MM. We consider here an approach proposed for a general problem of data fusion and one specifically intended to pansharpening. The method designed in [3] for the fusion of images acquired by heterogeneous sensors employs the analysis operator defined by Eq. 12 with the use of a square SE. More specifically, the author proposed the use of an undecimated approach, and thus the SE support has to be progressively larger for performing a multiresolution analysis of the image. Accordingly, the employed operator is given by

$$
T_{\mathrm{TO}}^{l}=\psi_{\mathrm{CO}, B^{l}}^{l}=\phi_{B^{l}} \gamma_{B^{l}},
$$

in which $B^{l}$ indicates a squared SE with side $2^{l}$. We tested this approach with both the HPF and HPM injection schemes defined by Eq. 2 and Eq. 3, as suggested in the cited paper [3] and in other works of the same author [40], [60]. We indicate this methods as MF-TO-HPF and MF-TO-HPM, respectively. The Top-Hat transform described by Eq. 14 was used in a MRA scheme based on HPF injection model in the work of Laporterie et al. [28], which was expressly devoted to pansharpening application. The downsampling was included in the decomposition operator that can thus be written as

$$
T_{\mathrm{LA}}^{l}=2^{\downarrow} \psi_{\mathrm{TH}, \mathrm{B}}=2^{\downarrow} 0.5\left(\phi_{B}+\gamma_{B}\right) .
$$

We use a linear-shaped $1 \times 2$ SE $B$ that was shown to represent the best option [28] and denote the algorithm as MF-LA.

Among the classical approaches we selected for each class three representative algorithms that achieve high performances (namely the best results) for most datasets [8]. With the inclusion of the pure upsampling scheme, denoted as EXP method, the seven employed algorithms were:

- EXP: MS image interpolation, using a polynomial kernel with 23 coefficients [20]

- PCA: Principal Component Analysis [39]

- GS: Gram Schmidt (Mode 1) [61]

- PRACS: Partial Replacement Adaptive Component Substitution [62]

- SFIM: Smoothing Filter-based Intensity Modulation [63], [64], based on High-Pass Modulation injection scheme and $5 \times 5$ box filter (i.e., mean filter) for details extraction

- AWLP: Additive Wavelet Luminance Proportional [56], using the 1-D "à trous" algorithm [59] based on the S\&M filter [58]

- MTF-GLP-HPM: GLP with MTF-matched filter [51] and HPF injection model [65]

Table II provides a synopsis of the numerical results, reporting the values of the algorithms' elaboration times and of the quality indexes related to both the reduced and full resolution assessments. In the following we analyze these results and report some remarks arising from the visual inspection of the fused products.

A first consideration can be drawn from the analysis of the computational burden, that can be derived by the first column of Table II. It reports the seconds required by the compared algorithms for completing the fusion of a PAN image and a 4channel MS image of size $512 \times 512 \mathrm{px}$ on an Intel $\AA$ Core $^{\mathrm{TM}} \mathrm{I} 7$ $3.2 \mathrm{GHz}$ processor. The approaches based on morphological 
TABLE II: QuANTitative RESUlts. For EACH DATASET, THE BEST RESUlt AMONG OPERATORS IS MARKED IN BOLD, THE SECOND ONE IS UNDERLINED AND THE THIRD IS WRITTEN IN ITALIC CHARACTERS.

\begin{tabular}{|c|c|c|c|c|c|c|c|c|c|c|c|c|}
\hline & & \multirow{3}{*}{$\begin{array}{l}\bar{n} \\
\tilde{\Xi} \\
\Xi\end{array}$} & \multicolumn{6}{|c|}{ Reduced resolution } & \multicolumn{4}{|c|}{ Full resolution } \\
\hline & & & \multicolumn{3}{|c|}{ Pleiades Dataset } & \multicolumn{3}{|c|}{ WV-2 Dataset } & \multicolumn{2}{|c|}{ Ikonos Dataset } & \multicolumn{2}{|c|}{ Geoeye Dataset } \\
\hline & & & Q4 & SAM & ERGAS & Q8 & SAM & ERGAS & SAM & SCC & SAM & SCC \\
\hline & EXP & 0 & 0.7782 & 4.6742 & 6.0826 & 0.7248 & 4.9263 & 5.4171 & 1.8568 & 0.4846 & 1.2561 & 0.5750 \\
\hline \multirow{3}{*}{ z } & PCA & 0.2 & 0.8122 & 6.1435 & 6.0028 & 0.8169 & 5.2153 & 4.4128 & 2.5136 & $\underline{0.9336}$ & 1.3533 & 0.9500 \\
\hline & GS & 0.1 & 0.8448 & 5.3304 & 5.0468 & 0.8335 & 4.8592 & 4.0144 & 2.4635 & $\overline{0.9354}$ & 1.3485 & 0.9502 \\
\hline & PRACS & 0.4 & 0.9325 & 4.5157 & 3.6523 & 0.8878 & 4.6678 & 3.6768 & 1.7454 & 0.9221 & 1.2575 & 0.8735 \\
\hline \multirow{3}{*}{ 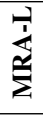 } & AWLP & 0.4 & 0.9426 & 4.3356 & 3.5219 & 0.9011 & 4.5146 & 3.3572 & 1.5710 & 0.9112 & 1.2514 & 0.9338 \\
\hline & SFIM & 0.1 & 0.9007 & 4.3599 & 4.2923 & 0.8758 & 4.2457 & 3.7591 & 1.7518 & 0.8600 & $\underline{1.2165}$ & 0.8906 \\
\hline & MTF-GLP-HPM & 0.2 & $\underline{0.9488}$ & $\underline{4.1541}$ & 3.2741 & 0.9092 & $\underline{3.8871}$ & 3.1005 & 1.4506 & 0.9120 & 1.2136 & 0.9506 \\
\hline \multirow{5}{*}{$\frac{\sum_{j}^{\prime}}{\sum}$} & MF-1 & 0.1 & 0.9271 & 4.2091 & 3.9832 & 0.8684 & 4.0964 & 4.0899 & 1.8057 & 0.7877 & 1.2755 & 0.9300 \\
\hline & MF-TO-HPF & 0.4 & 0.9207 & 4.5680 & 4.1687 & 0.8674 & 4.5915 & 4.1589 & 2.0149 & 0.8689 & 1.3165 & 0.8877 \\
\hline & MF-TO-HPM & 0.4 & 0.9194 & 4.4705 & 4.3820 & 0.8606 & 4.4041 & 4.8072 & 2.1155 & 0.7638 & 1.2888 & 0.8782 \\
\hline & MF-LA & 0.1 & 0.9390 & 4.2324 & 3.6285 & 0.8815 & 4.3085 & 3.7357 & 1.6095 & 0.9007 & 1.2576 & 0.9421 \\
\hline & MF-HG & 0.1 & 0.9503 & 4.0258 & $\underline{3.2933}$ & 0.9014 & $\mathbf{3 . 8 7 7 3}$ & $\underline{3.2884}$ & 1.3970 & 0.9108 & 1.2408 & 0.9499 \\
\hline
\end{tabular}

operators require an effort comparable to classical widespread algorithms, thus constituing a viable alternative for practical applications.

1) Reduced resolution assessment: The Pleiades and WV2 data sets are employed for this study and in both cases the proposed algorithm achieves remarkable results, often ranking between the first and the third position. It is clear that this novel approach represents a huge step forward with respect to existing methods based on MM.

More specifically, the Pleiades data set includes a synthetic PAN image generated by processing the MS channels, as described in Sect. IV-A. This procedure generates a narrow band synthetic PAN image and it has the advantage of not introducing a bias in the evaluation of the algorithms, as it happens for the optimal degradation procedure based on mimicking the sensor MTF. The target MS image, or Ground Truth (GT), of the Pleiades data set is reported in Fig. 5(a), showing a scene with many small objects. The results obtained by the MF-HG algorithm are remarkable since it scores the best in terms of quality indexes values. The visual analysis confirms the conclusions drawn from the quantitative evaluation. Thanks to the availability of the GT image, in the reduced resolution assessment procedure the visual inspection of the final product (portrayed in Fig. 5) can be performed by analyzing the injected details. In Fig. 6 the differences between the final products and the upsampled MS image $\widehat{\text { MS }}$ are reported. The comparison of the details injected by the tested algorithm (Fig. 6(b-f)) with the reference ones (Fig. 6(a)) evidences the balance between the spatial precision and the spectral fidelity that can be obtained by the proposed MF-HG method.

The WV2 data set provides supplementary information, due to the increasing number of channels. As expected, best results are achieved by the MRA methods, since a proper substitution of a single component is more difficult in this scenario. Also in this case the MF-HG attains appreciable results. In fact only the algorithms, whose detail extraction filter matches the filter used in the synthetic degradation procedure, yield higher values of the comprehensive $Q 8$ and $E R G A S$ indexes, while the best result in terms of spectral accuracy is still obtained by the MF-HG approach.

2) Full resolution assessment: The use of the full resolution assessment protocol is required for analyzing the performance of the algorithms at the effective working scale. However the lack of the GT makes more difficult the quantitative evaluation of the final products. Many indications derived at reduced resolution can be drawn also at full resolution. The two data sets represent completely different scenes, since the Ikonos data set (depicted in Fig. 3(c,g)) refers to an urban area characterized by many streets and building, namely by a large amount of geometric forms and contours. On the contrary the images composing the Geoeye data set (depicted in Fig. 3(d,h)) were acquired over a rural area and thus contain several objects with vague shape. In both tests the performances of the MF-HG algorithm are much better than those of the existing approaches based on morphological operators. Actually they are aligned with the results achieved by the best MRA schemes, as it can also be confirmed by a visual analysis of the extracted details that are represented in Fig. 7. Indeed the spectral accuracy is very high for both data sets. In terms of spatial correlation coefficient the method is outperformed only by the CS methods, that usually obtain greater SCC values due to the substitution of the whole intensity information in the final product. However, the overall performance of the MRA methods is surely more valuable, since the improvements of the spatial accuracy obtained by the CS approaches is paid with a significant loss of the spectral quality of the images, especially for the Ikonos data set.

By giving a closer look to the comparison among the MRAbased methods, it is evident that the proposed MF-HG method achieves performance very similar to the approaches that employ the knowledge of the sensor MTF, without requiring any information regarding the peculiar acquisition device. A more direct competitor is thus the AWLP that uses a fixed system (the S\&M filter) for carrying out the wavelet decomposition and that is outperformed by the proposed MF-HG approach. 


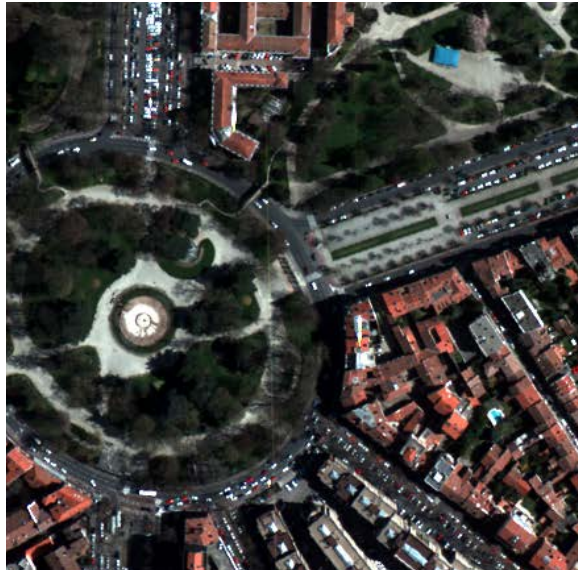

(a) GT

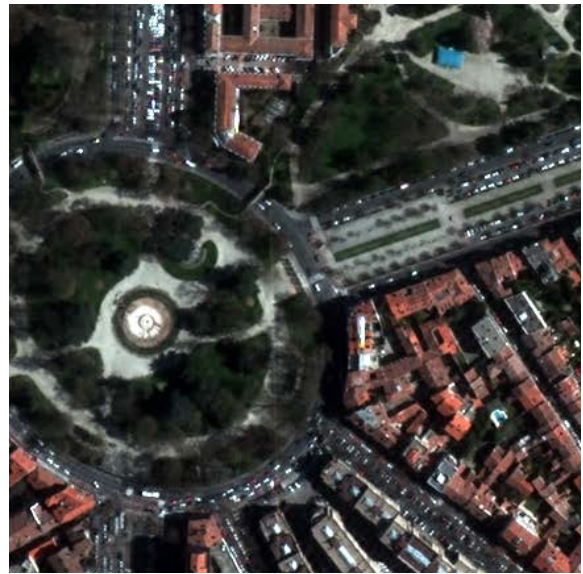

(d) MTF-GLP-HPM

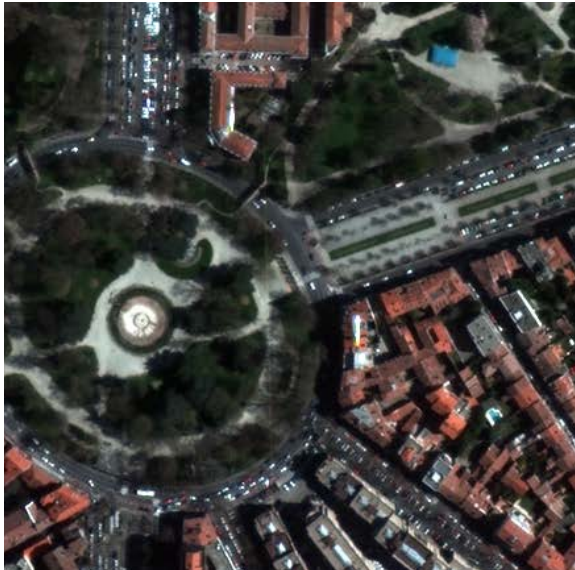

(b) PRACS

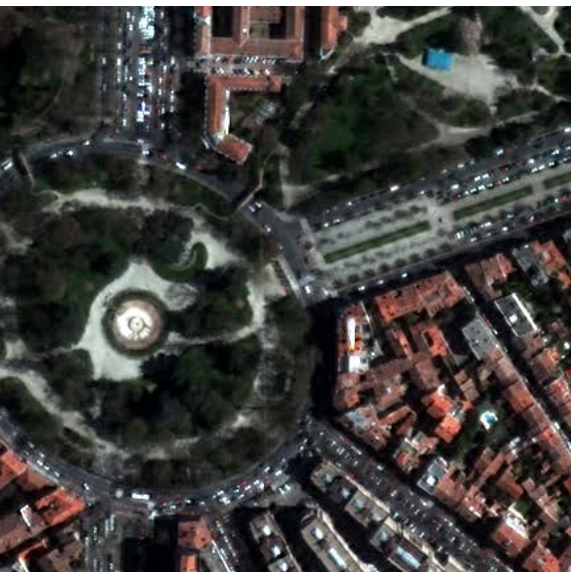

(e) MF-LA

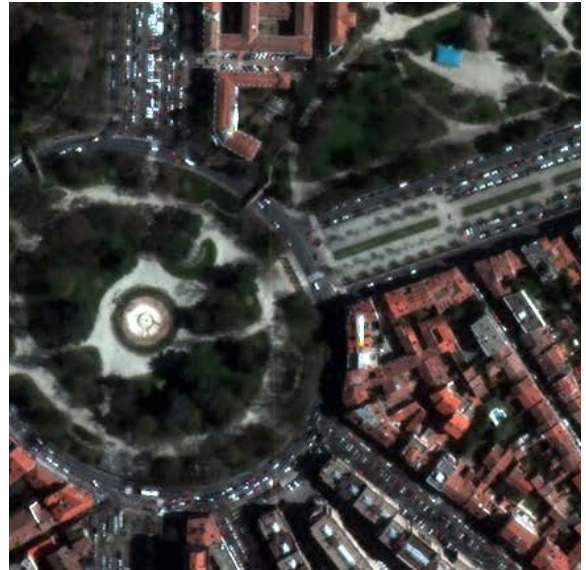

(c) AWLP

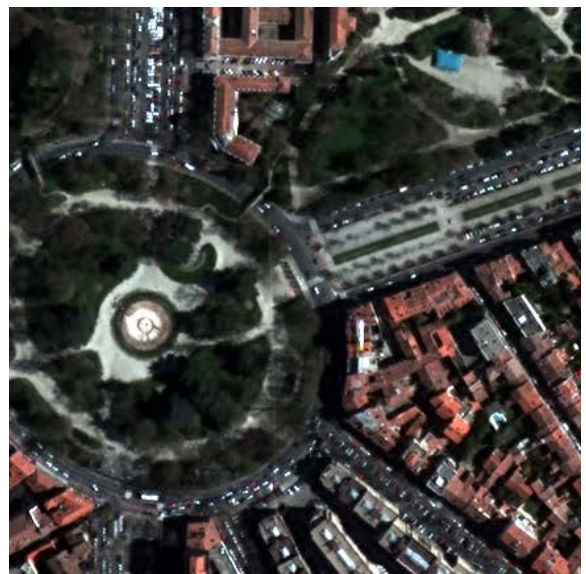

(f) MF-HG

Fig. 5: Final products of the pansharpening algorithms on the Pleiades data set: (a) GT; (b) BDSD; (c) AWLP; (d) MTF-GLPHPM; (e) MF-LA; (f) MF-HG.

\section{CONCLUSIONS}

In this work we studied the application of nonlinear image decomposition schemes based on morphological operators to data fusion, and in particular to the problem of pansharpening. Although the properties of morphology-based methods are widely exploited for applications as segmentation and denoising, only a limited number of data fusion approaches have taken advantage by their ability in dealing with shapes.

The effective application of MM to pansharpening requires the choice of a suitable spatial detail extraction operator that we designed as the difference of the two half-gradients. We evidenced that it allows to highlight all the spatial changes of the input image, preserving the dynamics of the signal variation and a local zero mean value, as required by pansharpening applications. A comprehensive fusion architecture, encompassing the choice of the MRA implementation options and of the detail injection method was here proposed and evaluated. Four data sets acquired by four different sensors were used for the algorithm assessment, using both the reduced and full resolution quality evaluation protocols.

The results show that the proposed method greatly outclasses the existing pansharpening algorithms based on mathematical morphology. Actually it achieves top-class perfor- mance, aligned with that of best pansharpening algorithms, without requiring specific knowledge of the used sensor, as for example the MTF shape.

As possible future developments, we plan to consider other tools provided by mathematical morphology that can be suitable in data fusion problems, as for example the levelings operators.

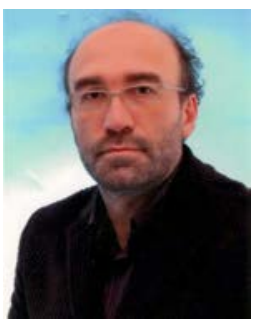

Rocco Restaino (M'14) received the Laurea degree in electronic engineering from the University of Naples, Naples, Italy, in 1998, and the Ph.D. degree in information engineering from the University of Salerno, Italy, in 2002. He is currently an Assistant Professor at the University of Salerno. His research interests include probability theory, stochastic geometry and signal processing for remote sensing and networking. He was awarded with the IGARSS 2015 Symposium Best Paper Award. 


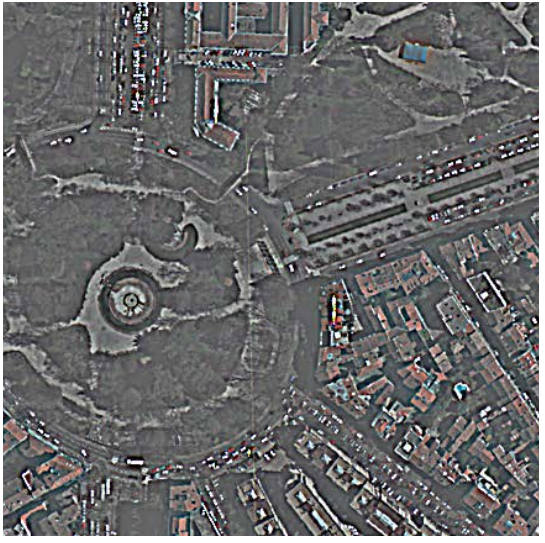

(a) GT

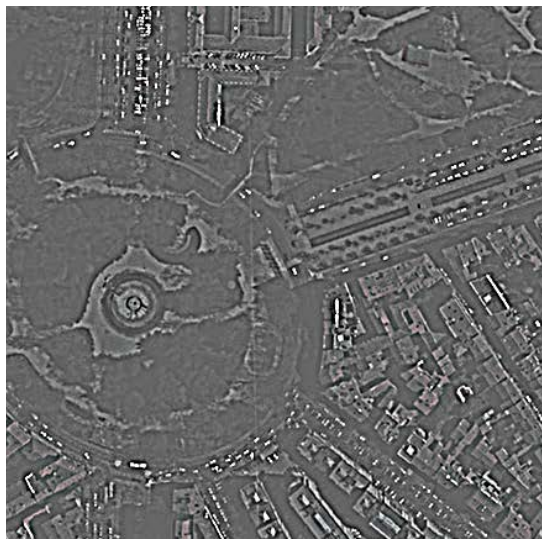

(d) MTF-GLP-HPM

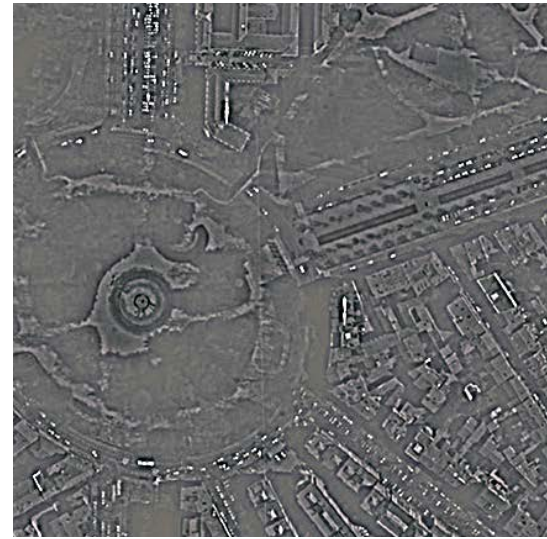

(b) PRACS

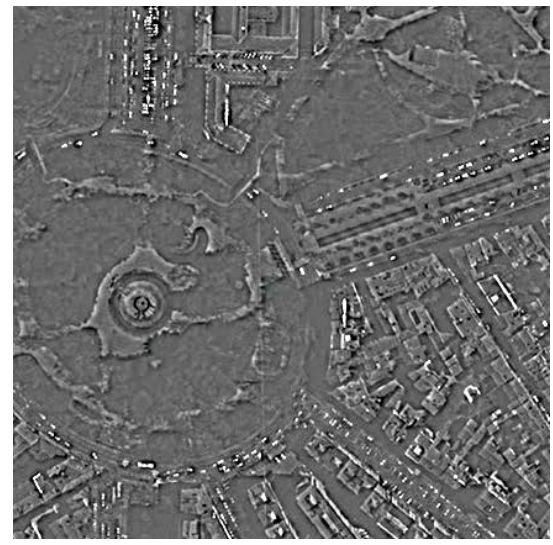

(e) MF-LA

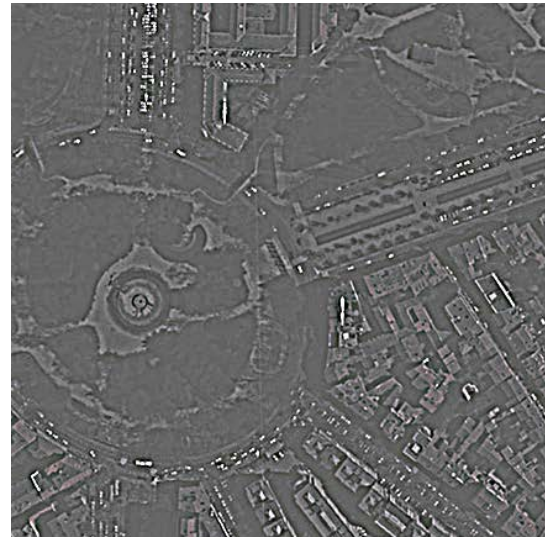

(c) AWLP

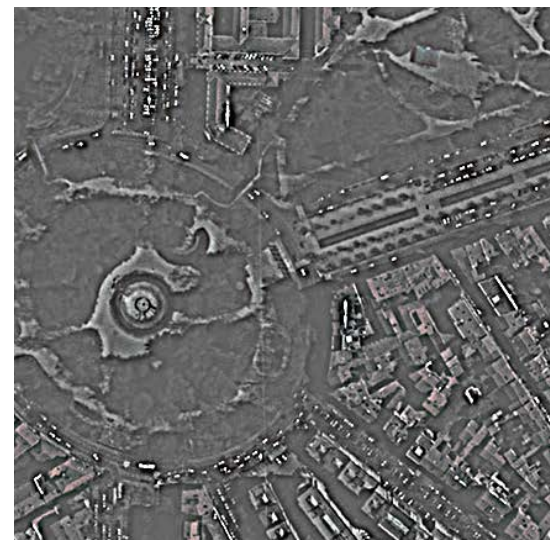

(f) $\mathrm{MF}-\mathrm{HG}$

Fig. 6: Details extracted by the pansharpening algorithms on the Pleiades data set: (a) GT; (b) BDSD; (c) AWLP; (d) MTFGLP-HPM; (e) MF-LA; (f) MF-HG.

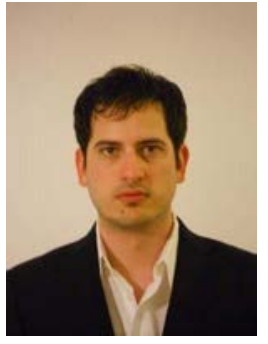

Gemine Vivone received the B.Sc. (cum laude), the M.Sc. (cum laude), and the Ph.D. degrees in information engineering from the University of Salerno, Salerno, Italy, in 2008, 2011, and 2014, respectively. $\mathrm{He}$ is currently a Scientist at the North Atlantic Treaty Organization (NATO) Science \& Technology Organization (STO) Centre for Maritime Research and Experimentation (CMRE), La Spezia, Italy. In 2014, he joined the NATO STO CMRE, La Spezia, Italy as a Research Fellow. In 2013, he was as a Visiting Scholar with Grenoble Institute of Technology (INPG), Grenoble, France, conducting his research at the Laboratoire Grenoblois de IImage, de la Parole, du Signal et de 1Automatique GIPSALab. In 2012, he was a Visiting Researcher with the NATO Undersea Research Centre, La Spezia, Italy. His main research interests focus on statistical signal processing, detection of remotely sensed images, data fusion, and tracking algorithms. Dr. Vivone serves as a Referee for several journals, such as IEEE Transactions on Geoscience and Remote Sensing, IEEE Journal of Selected Topics in Applied Earth Observations and Remote Sensing, and IEEE Geoscience and Remote Sensing Letters. Dr. Vivone was the recipient of the Symposium Best Paper Award at the IEEE International Geoscience and Remote Sensing Symposium (IGARSS) 2015.

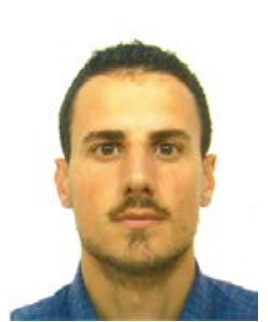

Mauro Dalla Mura (S'08 - M'11) received the laurea (B.E.) and laurea specialistica (M.E.) degrees in Telecommunication Engineering from the University of Trento, Italy, in 2005 and 2007, respectively. He obtained in 2011 a joint $\mathrm{Ph} . \mathrm{D}$. degree in Information and Communication Technologies (Telecommunications Area) from the University of Trento, Italy and in Electrical and Computer Engineering from the University of Iceland, Iceland. In 2011 he was a Research fellow at Fondazione Bruno Kessler, Trento, Italy, conducting research on computer vision.

He is currently an Assistant Professor at Grenoble Institute of Technology (Grenoble INP), France. He is conducting his research at the Grenoble Images Speech Signals and Automatics Laboratory (GIPSA-Lab). His main research activities are in the fields of remote sensing, image processing and pattern recognition. In particular, his interests include mathematical morphology, classification and multivariate data analysis.

Dr. Dalla Mura was the recipient of the IEEE GRSS Second Prize in the Student Paper Competition of the 2011 IEEE IGARSS 2011 and co-recipient of the Best Paper Award of the International Journal of Image and Data Fusion for the year 2012-2013 and the Symposium Paper Award for IEEE IGARSS 2014.

He is the President of the IEEE GRSS French Chapter for the period 20162019 (he served as Secretary in 2013-2016). 


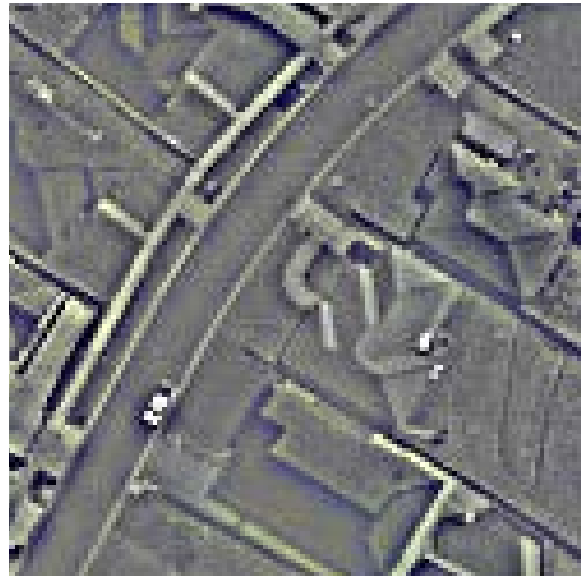

(a) PRACS

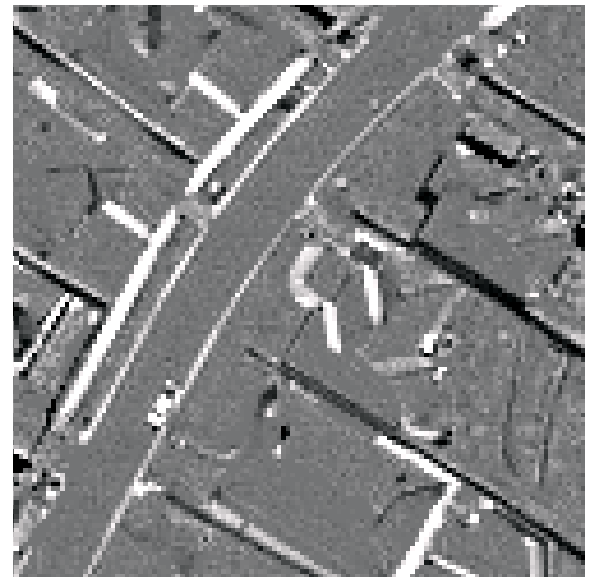

(d) MF-TO-HPM

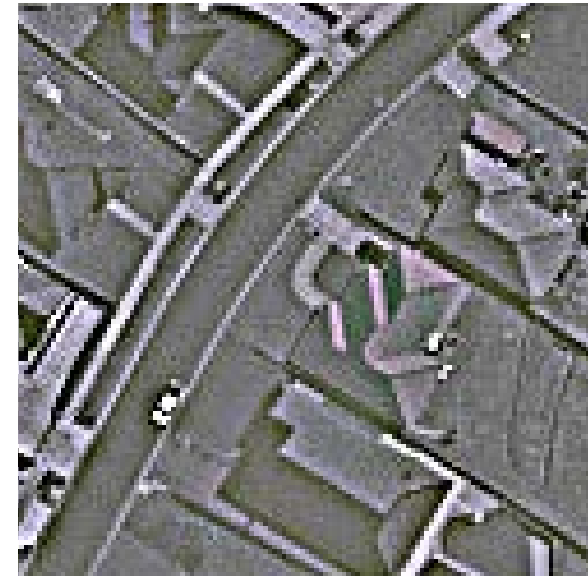

(b) AWLP

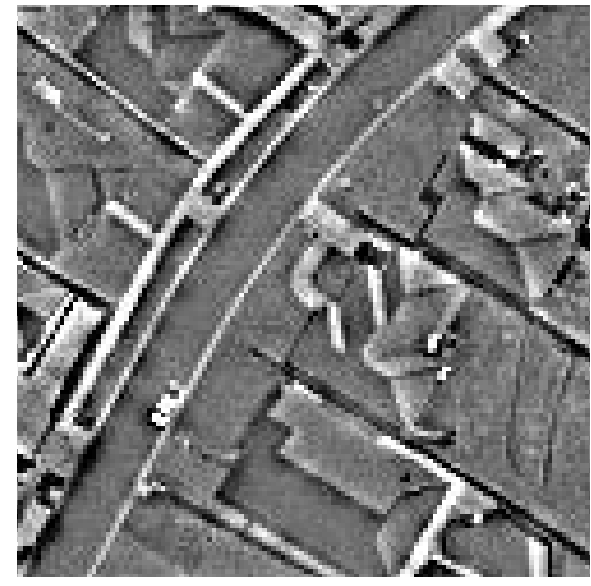

(e) MF-LA

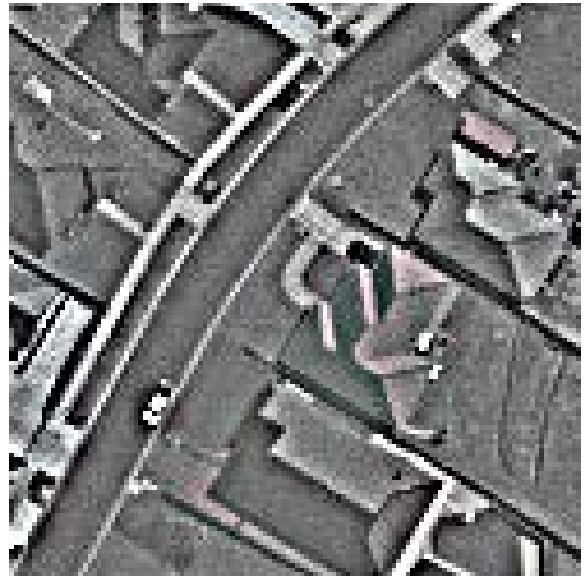

(c) MTF-GLP-HPM

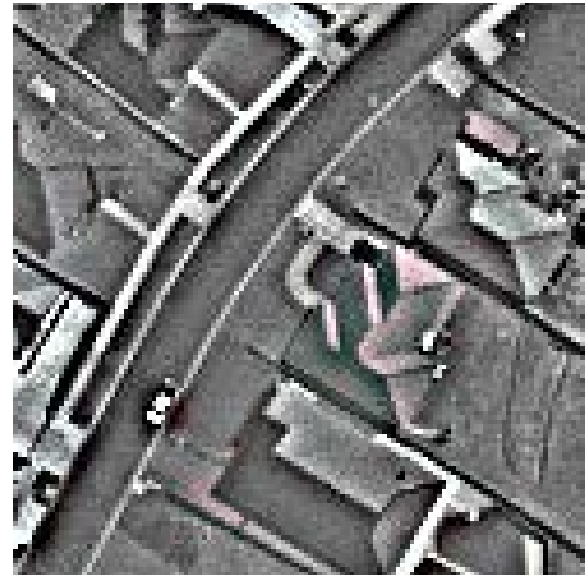

(f) MF-HG

Fig. 7: Details extracted by the pansharpening algorithms on the Geoeye data set: (a) BDSD; (b) AWLP; (c) MTF-GLP-HPM; (d) MF-TO-HPM; (e) MF-LA; (f) MF-HG.

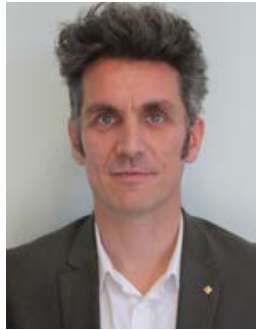

Jocelyn Chanussot (M'04SM'04F'12) received the M.Sc. degree in electrical engineering from the Grenoble Institute of Technology (Grenoble INP), Grenoble, France, in 1995, and the Ph.D. degree from Savoie University, Annecy, France, in 1998. In 1999, he was with the Geography Imagery Perception Laboratory for the Delegation Generale de l'Armement (DGA - French National Defense Department). Since 1999, he has been with Grenoble INP, where he was an Assistant Professor from 1999 to 2005, an Associate Professor from 2005 to 2007, and is currently a Professor of signal and image processing. He is conducting his research at the Grenoble Images Speech Signals and Automatics Laboratory (GIPSA-Lab). His research interests include image analysis, multicomponent image processing, nonlinear filtering, and data fusion in remote sensing. He has been a visiting scholar at Stanford University (USA), KTH (Sweden) and NUS (Singapore). Since 2013, he is an Adjunct Professor of the University of Iceland. In 2015-2017, he is a visiting professor at the University of California, Los Angeles (UCLA). Dr. Chanussot is the founding President of IEEE Geoscience and Remote Sensing French chapter (20072010) which received the 2010 IEEE GRS-S Chapter Excellence Award. He was the co-recipient of the NORSIG 2006 Best Student Paper Award, the IEEE GRSS 2011 and 2015 Symposium Best Paper Award, the IEEE GRSS 2012 Transactions Prize Paper Award and the IEEE GRSS 2013 Highest Impact Paper Award. He was a member of the IEEE Geoscience and Remote Sensing Society AdCom (2009-2010), in charge of membership development. He was the General Chair of the first IEEE GRSS Workshop on Hyperspectral Image and Signal Processing, Evolution in Remote sensing (WHISPERS). He was the Chair (2009-2011) and Cochair of the GRS Data Fusion Technical Committee (2005-2008). He was a member of the Machine Learning for
Signal Processing Technical Committee of the IEEE Signal Processing Society (2006-2008) and the Program Chair of the IEEE International Workshop on Machine Learning for Signal Processing, (2009). He was an Associate Editor for the IEEE Geoscience and Remote Sensing Letters (2005-2007) and for Pattern Recognition (2006-2008). Since 2007, he is an Associate Editor for the IEEE Transactions on Geoscience and Remote Sensing. He was the Editor-inChief of the IEEE Journal of Selected Topics in Applied Earth Observations and Remote Sensing (2011-2015). In 2013, he was a Guest Editor for the Proceedings of the IEEE and in 2014 a Guest Editor for the IEEE Signal Processing Magazine. He is a Fellow of the IEEE and a member of the Institut Universitaire de France (2012-2017).

\section{REFERENCES}

[1] J. Serra, Image Analysis and Mathematical Morphology. Academic Press, 1984

[2] P. Soille, Morphological Image Analysis: Principles and Applications. Springer-Verlag, 2003.

[3] A. Toet, "Hierarchical image fusion," Mach. Vision App., vol. 3, no. 1, pp. $1-11,1990$

[4] S. Mukhopadhyay and B. Chanda, "Fusion of 2D grayscale images using multiscale morphology," Pattern Recogn., vol. 34, no. 10, pp. 19391949, 2001.

[5] X. Bai, "Morphological image fusion using the extracted image regions and details based on multi-scale top-hat transform and toggle contrast operator," Digit. Signal Process., vol. 23, no. 2, pp. 542-554, Mar. 2013.

[6] M. Dalla Mura, S. Prasad, F. Pacifici, P. Gamba, J. Chanussot, and J. A. Benediktsson, "Challenges and opportunities of multimodality and data fusion in remote sensing," Proc. IEEE, vol. 103, no. 9, pp. 1585-1601, Sept 2015 . 
[7] B. Aiazzi, L. Alparone, S. Baronti, A. Garzelli, and M. Selva, "Twentyfive years of pansharpening: A critical review and new developments," in Signal and Image Processing for Remote Sensing, 2nd ed., C.-H. Chen, Ed. Boca Raton, FL, USA: CRC Press, 2012, pp. 533-548.

[8] G. Vivone, L. Alparone, J. Chanussot, M. D. Mura, A. Garzelli, G. Licciardi, R. Restaino, and L. Wald, "A critical comparison among pansharpening algorithms," IEEE Trans. Geosci. Remote Sens., vol. 53, no. 5, pp. 2565-2586, May 2015.

[9] S. Li and B. Yang, "A new pan-sharpening method using a compressed sensing technique," IEEE Trans. Geosci. Remote Sens., vol. 49, no. 2, pp. 738-746, Feb. 2011.

[10] X. He, L. Condat, J. Bioucas-Dias, J. Chanussot, and J. Xia, "A new pansharpening method based on spatial and spectral sparsity priors," IEEE Trans. Image Process., vol. 23, no. 9, pp. 4160-4174, Sep. 2014.

[11] M. R. Vicinanza, R. Restaino, G. Vivone, M. Dalla Mura, G. Licciardi, and J. Chanussot, "A pansharpening method based on the sparse representation of injected details," IEEE Geosci. Remote Sens. Lett., vol. 12 , no. 1, pp. 180-184, Jan. 2015.

[12] D. Fasbender, J. Radoux, and P. Bogaert, "Bayesian data fusion for adaptable image pansharpening," IEEE Trans. Geosci. Remote Sens., vol. 46, no. 6, pp. 1847-1857, Jun. 2008.

[13] C. Ballester, V. Caselles, L. Igual, J. Verdera, and B. Rougé, "A variational model for $\mathrm{P}+\mathrm{XS}$ image fusion," Int. J. Comput. Vision, vol. 69, no. 1, pp. 43-58, Aug. 2006.

[14] L. Alparone, B. Aiazzi, S. Baronti, and A. Garzelli, Remote Sensing Image Fusion. CRC Press, 2015.

[15] L. Alparone, L. Wald, J. Chanussot, C. Thomas, P. Gamba, and L. M. Bruce, "Comparison of pansharpening algorithms: Outcome of the 2006 GRS-S data fusion contest," IEEE Trans. Geosci. Remote Sens., vol. 45, no. 10, pp. 3012-3021, Oct. 2007.

[16] S. Baronti, B. Aiazzi, M. Selva, A. Garzelli, and L. Alparone, "A theoretical analysis of the effects of aliasing and misregistration on pansharpened imagery," IEEE J. Sel. Topics Signal Process., vol. 5, no. 3, pp. 446-453, Jun. 2011.

[17] L. Amolins, Y. Zhang, and P. Dare, "Wavelet based image fusion techniques - An introduction, review and comparison," ISPRS J. Photogramm. Remote Sens., vol. 62, no. 4, pp. 249-263, Sep. 2007.

[18] L. Bin, M. M. Khan, T. Bienvenu, and J. Chanussot, "Context-adaptive pansharpening based on binary partition tree segmentation," in Proc. ICIP, 2010, pp. 1977-1980.

[19] F. Nencini, A. Garzelli, S. Baronti, and L. Alparone, "Remote sensing image fusion using the curvelet transform," Inform. Fusion, vol. 8, no. 2, pp. 143-156, Sep. 2007.

[20] B. Aiazzi, L. Alparone, S. Baronti, and A. Garzelli, "Context-driven fusion of high spatial and spectral resolution images based on oversampled multiresolution analysis," IEEE Trans. Geosci. Remote Sens., vol. 40, no. 10, pp. 2300-2312, Oct. 2002.

[21] J. Goutsias and H. J. A. M. Heijmans, "Nonlinear multiresolution signal decomposition schemes. I. morphological pyramids," IEEE Trans. Image Process., vol. 9, no. 11, pp. 1862-1876, Nov. 2000.

[22] — "Nonlinear multiresolution signal decomposition schemes. II. morphological wavelets," IEEE Trans. Image Process., vol. 9, no. 11, pp. 1897-1913, Nov. 2000.

[23] J.-L. Starck, F. Murtagh, and J. M. Fadili, Sparse image and signal processing: wavelets, curvelets, morphological diversity. Cambridge University Press, 2010.

[24] L. A. Overturf, M. L. Comer, and E. J. Delp, "Color image coding using morphological pyramid decomposition," IEEE Trans. Image Process., vol. 4, no. 2, pp. 177-185, Feb. 1995.

[25] J. B. T. M. Roerdink, "Multiresolution maximum intensity volume rendering by morphological adjunction pyramids," IEEE Trans. Image Process., vol. 12, no. 6, pp. 653-660, Jun. 2003.

[26] G. K. Matsopoulos, S. Marshall, and J. N. H. Brunt, "Multiresolution morphological fusion of MR and CT images of the human brain," IEE P-Vis. Image Sign., vol. 141, no. 3, pp. 137-142, Jun. 1994.

[27] G. K. Matsopoulos and S. Marshall, "Application of morphological pyramids: Fusion of MR and CT phantoms," J. Vis. Commun. Image Represent., vol. 6, no. 2, pp. 196-207, Jun. 1995.

[28] F. Laporterie-Déjean, O. Amram, G. Flouzat, E. Pilicht, and M. Gayt, "Data fusion thanks to an improved morphological pyramid approach: comparison loop on simulated images and application to SPOT 4 data"," in Proc. IEEE IGARSS, 2000, pp. 2117-2119.

[29] F. Laporterie-Déjean, G. Flouzat, and O. Amram, "Mathematical morphology multi-level analysis of trees patterns in savannas," in Proc. IEEE IGARSS, 2001, pp. 1496-1498.
[30] S. I. Bejinariu, F. Rotaru, C. D. Niţă, and M. Costin, "Morphological wavelets for panchromatic and multispectral image fusion," in Soft Computing Applications. Springer, 2013, pp. 573-583.

[31] R. Restaino, G. Vivone, M. Dalla Mura, and J. Chanussot, "A pansharpening algorithm based on morphological filters," in Mathematical Morphology and Its Applications to Signal and Image Processing, J. A. Benediktsson, J. Chanussot, L. Najman, and H. Talbot, Eds. Springer, 2015, pp. 98-109.

[32] P. Maragos and R. W. Schafer, "Morphological filters-part I: Their settheoretic analysis and relations to linear shift-invariant filters," IEEE Trans. Acoust. Speech Signal Process., vol. 35, no. 8, pp. 1153-1169, Aug. 1987.

[33] — "Morphological filters-part II: Their relations to median, orderstatistic, and stack filters," IEEE Trans. Acoust. Speech Signal Process., vol. 35, no. 8, pp. 1170-1184, Aug. 1987.

[34] R. Stevenson and G. R. Arce, "Morphological filters: Statistics and further syntactic properties," IEEE Trans. Circuits Syst., vol. 34, no. 11, pp. 1292-1305, Nov. 1987

[35] R. A. Schowengerdt, Remote Sensing: Models and Methods for Image Processing, 2nd ed. Orlando, FL, USA: Academic Press, 1997.

[36] G. Vivone, R. Restaino, M. Dalla Mura, G. Licciardi, and J. Chanussot, "Contrast and error-based fusion schemes for multispectral image pansharpening," IEEE Geosci. Remote Sens. Lett., vol. 11, no. 5, pp. 930-934, May 2014.

[37] E. Peli, "Contrast in complex images," J. Opt. Soc. Amer. A, vol. 7, no. 10, pp. 2032-2039, Oct. 1990.

[38] G. R. Arce and S. A. Fontana, "On the midrange estimator," IEEE Trans. Acoust. Speech Signal Process., vol. 1988, no. 1, pp. 920-922, Jun. 1988.

[39] P. S. Chavez, Jr., S. C. Sides, and J. A. Anderson, "Comparison of three different methods to merge multiresolution and multispectral data: Landsat TM and SPOT panchromatic," Photogramm. Eng. Remote Sens., vol. 57, no. 3, pp. 295-303, Mar. 1991.

[40] A. Toet, "A morphological pyramidal image decomposition," Pattern Recogn. Lett., vol. 9, no. 4, pp. 255-261, 1989.

[41] M. A. Schulze and J. A. Pearce, "Linear combinations of morphological operators: the midrange, pseudomedian, and LOCO filters," in Proc. IEEE ICASSP, vol. 5, 1993, pp. 57-60.

[42] G. Flouzat, O. Amram, F. Laporterie-Déjean, and S. Cherchali, "Multiresolution analysis and reconstruction by a morphological pyramid in the remote sensing of terrestrial surfaces," Signal Process., vol. 81, no. 10 , pp. 2171-2185, Oct. 2001.

[43] F. Laporterie-Déjean, G. Flouzat, and O. Amram, "The morphological pyramid and its applications to remote sensing: Multiresolution data analysis and features extraction," Image Anal. Stereol., vol. 21, no. 1, pp. 49-53, 2002.

[44] X. Bai, F. Zhou, and B. Xue, "Edge preserved image fusion based on multiscale toggle contrast operator," Image Vision Comput., vol. 29, no. 12, pp. 829-839, 2011.

[45] J.-L. Starck, F. Murtagh, B. Pirenne, and M. Albrecht, "Astronomical image compression based on noise suppression," Publ. Astron. Soc. Pac., vol. 108, no. 723, pp. 446-455, May 1996.

[46] A. R. Jiménez-Sánchez, J. D. Mendiola-Santibañez, I. R. TerolVillalobos, G. Herrera-Ruíz, D. Vargas-Vázquez, J. J. García-Escalante, and A. Lara-Guevara, "Morphological background detection and enhancement of images with poor lighting," IEEE Trans. Image Process., vol. 18, no. 3, pp. 613-623, Mar. 2009.

[47] E. L. Crow and M. M. Siddiqui, "Robust estimation of location," J. Am. Statist. Assoc., vol. 62, no. 318, pp. 353-389, Jun. 1967.

[48] H. L. Harter, "The method of least squares and some alternatives: Part iv," Int. Stat. Rev., vol. 43, no. 2, pp. 125-190, Aug. 1975.

[49] P. R. Rider, "The midrange of a sample as an estimator of the population midrange," J. Am. Statist. Assoc., vol. 52, no. 280, pp. 537-542, 2005.

[50] L. Wald, T. Ranchin, and M. Mangolini, "Fusion of satellite images of different spatial resolutions: Assessing the quality of resulting images," Photogramm. Eng. Remote Sens., vol. 63, no. 6, pp. 691-699, Jun. 1997.

[51] B. Aiazzi, L. Alparone, S. Baronti, A. Garzelli, and M. Selva, "MTFtailored multiscale fusion of high-resolution MS and Pan imagery," Photogramm. Eng. Remote Sens., vol. 72, no. 5, pp. 591-596, May 2006.

[52] R. H. Yuhas, A. F. H. Goetz, and J. W. Boardman, "Discrimination among semi-arid landscape endmembers using the Spectral Angle Mapper (SAM) algorithm," in Proc. Summaries 3rd Annu. JPL Airborne Geosci. Workshop, 1992, pp. 147-149.

[53] L. Alparone, S. Baronti, A. Garzelli, and F. Nencini, "A global quality measurement of pan-sharpened multispectral imagery," IEEE Trans. Geosci. Remote Sens., vol. 1, no. 4, pp. 313-317, Oct. 2004. 
[54] A. Garzelli and F. Nencini, "Hypercomplex quality assessment of multi/hyper-spectral images," IEEE Trans. Geosci. Remote Sens., vol. 6, no. 4, pp. 662-665, Oct. 2009.

[55] L. Wald, Data Fusion: Definitions and Architectures - Fusion of images of different spatial resolutions. Paris, France: Les Presses de l'École des Mines, 2002.

[56] X. Otazu, M. González-Audícana, O. Fors, and J. Núñez, "Introduction of sensor spectral response into image fusion methods. Application to wavelet-based methods," IEEE Trans. Geosci. Remote Sens., vol. 43, no. 10, pp. 2376-2385, Oct. 2005.

[57] J. Canny, "A computational approach to edge detection," IEEE Trans. Pattern Anal. Mach. Intell., vol. 8, no. 6, pp. 679-698, Nov. 1986.

[58] G. Strang and T. Nguyen, Wavelets and Filter Banks, 2nd ed. Wellesley, MA: Wellesley Cambridge Press, 1996.

[59] J.-L. Starck, J. Fadili, and F. Murtagh, "The undecimated wavelet decomposition and its reconstruction," IEEE Trans. Image Process., vol. 16, no. 2, pp. 297-309, Feb. 2007.

[60] A. Toet, "Image fusion by a ratio of low pass pyramid," Pattern Recognition Letters, vol. 9, no. 4, pp. 245-253, May 1989.

[61] C. A. Laben and B. V. Brower, "Process for enhancing the spatial resolution of multispectral imagery using pan-sharpening," 2000, U.S. Patent \# 6,011,875.

[62] J. Choi, K. Yu, and Y. Kim, "A new adaptive component-substitutionbased satellite image fusion by using partial replacement," IEEE Trans. Geosci. Remote Sens., vol. 49, no. 1, pp. 295-309, Jan. 2011.

[63] J. G. Liu, "Smoothing filter based intensity modulation: A spectral preserve image fusion technique for improving spatial details," Int. J. Remote Sens., vol. 21, no. 18, pp. 3461-3472, Dec. 2000.

[64] L. Wald and T. Ranchin, "Comment: Liu 'Smoothing filter-based intensity modulation: A spectral preserve image fusion technique for improving spatial details'," Int. J. Remote Sens., vol. 23, no. 3, pp. $593-$ 597, Jan. 2002.

[65] B. Aiazzi, L. Alparone, S. Baronti, A. Garzelli, and M. Selva, "An MTF-based spectral distortion minimizing model for pan-sharpening of very high resolution multispectral images of urban areas," in Proc. 2nd GRSS/ISPRS Joint Workshop Remote Sens. Data Fusion URBAN Areas, 2003, pp. 90-94. 